\title{
AVALIAÇÃO DE CLORETOS NÃO TAMPONADOS COMO EXTRATORES DE ALUMÍNIO ASSOCIADO À MATÉRIA ORGÂNICA EM SOLOS DA PLANÍCIE COSTEIRA DO ESTADO DE SÃO PAULO ${ }^{(1)}$
}

\author{
Maurício Rizzato Coelho ${ }^{(2)}$, Pablo Vidal-Torrado ${ }^{(3)}$, Xosé Luis Otero \\ Pérez ${ }^{(4)}$, Vanda Moreira Martins ${ }^{(5)}$ \& Felipe Macías Vázquez ${ }^{(4)}$
}

\begin{abstract}
RESUMO
Pirofosfato de sódio é o extrator mais utilizado para a determinação do $\mathrm{Al}$ complexado à matéria orgânica do solo (MOS). Devido à sua falta de seletividade para algumas amostras de solos, extratores não tamponados de $\mathrm{Cl}$ têm sido recomendados em substituição ao pirofosfato. Com o objetivo de avaliar a eficácia dos cloretos não tamponados de $\mathrm{Cu}\left(\mathrm{CuCl}_{2}\right)$, lantânio $\left(\mathrm{LaCl}_{3}\right)$ e potássio $(\mathrm{KCl})$ como extratores de $\mathrm{Al}$ unido à MOS, analisaram-se amostras de 31 perfis de solos representativos das áreas sob vegetação de restinga do litoral paulista. Os resultados foram comparados àqueles obtidos com pirofosfato de sódio. $\mathrm{O} \mathrm{CuCl}_{2}$, mais forte que o $\mathrm{LaCl}_{3}$ e $\mathrm{KCl}$ e menos eficaz que o pirofosfato, extraiu parte do $\mathrm{Al}$ de compostos inorgânicos amorfos e de complexos Al-húmus bastante estáveis; estes últimos possivelmente não são reativos em termos de acidez do solo e troca iônica. Ao contrário, $\mathrm{KCl}$ e $\mathrm{LaCl}_{3}$ removeram somente as formas reativas do elemento associadas ao C orgânico do solo (Corg). No entanto, o $\mathrm{Al}$ extraído por $\mathrm{LaCl}_{3}\left(\mathrm{Al}_{\mathrm{La}}\right)$ foi que melhor se correlacionou com o Corg e com a CTC, indicando que $\mathrm{Al}_{\mathrm{La}}$ está relacionado ao principal componente responsável pelo desenvolvimento de cargas nos solos estudados. Os extratores permitiram analisar o grau de interação da MOS com o Al e a influência do pH do solo na interação. Os complexos Al-húmus
\end{abstract}

\footnotetext{
(1) Parte da Tese de Doutorado do primeiro autor apresentada à Escola Superior de Agricultura "Luiz de Queiroz" - ESALQ/USP, realizada com o auxílio da CAPES e FAPESP. Recebido para publicação em 17 de agosto de 2010 e aprovado em 14 de julho de 2011.

(2) Pesquisador do Centro Nacional de Pesquisa em Solos da Embrapa Solos. Rua Jardim Botânico 1024. CEP 22460-000 Jardim Botânico, Rio de Janeiro (RJ). E-mail: mauricio@cnps.embrapa.br

(3) Professor do Departamento de Ciência do Solo, Escola Superior de Agricultura "Luiz de Queiroz" - ESALQ/USP. Caixa Postal 09, CEP 13418-900 Piracicaba (SP). Bolsista do CNPq. E-mail: pablo@esalq.usp.br

(4) Professor do Departamento de Edafología de la Universidad de Santiago de Compostela. Campus Universitario Sur. Faculdad de Bioloxía. Espanha. E-mails: xl.otero@usc.es; felipe.macias.vazquez@usc.es

(5) Professora do Curso de Geografia da Universidade Estadual do Oeste do Paraná - UNIOESTE. Campus de Marechal Cândido Rondon. Rua Pernambuco 1777, Centro, CEP 85960-000 Marechal Cândido Rondon (PR). E-mail: mmvanda@hotmail.com
} 
mais estáveis foram observados nos horizontes bem drenados Bs, Bhs e C, em que o $\mathrm{LaCl}_{3}$ e, sobretudo, o $\mathrm{KCl}$ mostraram as mais baixas eficácias entre todas as amostras analisadas. O Al mais lábil unido à MOS foi encontrado nos horizontes superficiais do tipo A. De maneira geral, os horizontes estudados apresentaram a seguinte sequência de estabilidade da interação Al-húmus: $\mathrm{A}<\mathrm{Bh} \leq \mathrm{C}<\mathrm{Bhm}<\mathrm{Bs} /$ Bhs/Bsm. Esses últimos, juntamente com alguns poucos horizontes $C$, são os únicos horizontes de subsuperfície bem drenados, indicando que o hidromorfismo nos horizontes $\mathrm{Bh}$ e a ciclagem da serapilheira nos horizontes A favorecem a manutenção de espécies de alumínio mais lábeis nos solos estudados.

Termos de indexação: Espodossolos, pirofosfato de sódio, $\mathrm{CuCl}_{2} ; \mathrm{LaCl}_{3}, \mathrm{KCl}$, complexos Al-húmus.

\title{
SUMMARY: EVALUATION OF UNBUFFERED CHLORIDE TO EXTRACT ALUMINUM BOUND TO ORGANIC MATTER FROM SOILS OF THE COASTAL PLAIN OF SÃO PAULO STATE
}

\begin{abstract}
Sodium pyrophosphate is the most widely used extractant of Al bound to soil organic matter (Al-humus). However, its selectivity has been questioned, so that some authors have proposed the use of substitutional extractants. To evaluate the effectiveness of unbuffered $\mathrm{Cu}$ $\left(\mathrm{CuCl}_{2}\right), \mathrm{La}\left(\mathrm{LaCl}_{3}\right)$ and $\mathrm{K}(\mathrm{KCl})$ chlorides to extract Al-humus, soil material from 31 representative pedons of Spodosols and Quartzipsamments from sandy coastal plains of São Paulo State was evaluated. The results were compared with those achieved by sodium pyrophosphate. $\mathrm{CuCl}_{2}$ was more efficient than $\mathrm{LaCl}_{3}$ and $\mathrm{KCl}$ and $\mathrm{LaCl}_{3}$ less than pyrophosphate; i.e., extracted part of the Al associated to inorganic poorly crystalline compounds and stable organic complexes, the latter possibly inactive in terms of soil acidity and ionic exchange. On the other hand, $\mathrm{KCl}$ and $\mathrm{LaCl}_{3}$ removed the reactive Al forms. However, $\mathrm{Al}$ extracted by $\mathrm{LaCl}_{3}\left(\mathrm{Al}_{\mathrm{La}}\right)$ was best correlated with organic carbon (Corg) and soil CEC, indicating that $A l_{L a}$ is related to the main component responsible for the development of charges in the studied soil. The extractors allowed analyzing the Al-humus interaction degree, as well as the influence of soil $\mathrm{pH}$ in this interaction. The most stable Al-humus complexes were only found in the well-drained Bs, Bhs and C horizons, where the lowest effectiveness of all soil materials analyzed was found for $\mathrm{LaCl}_{3}$ and mainly $\mathrm{KCl}$. The more labile organic matter bounded Al was found in the A horizons. In general, the stability of Al-humus interaction of the studied horizons showed the following sequence: $A<B h \leq C<B h m<B s / B h s / B s m$, of which the latter and a few $C$ are the only well-drained subsurface horizons, indicating that the presence of water (Bh horizons) and litter turnover (A horizons) are favorable for the maintenance of the more labile Al species.
\end{abstract}

Index terms: Spodosols; sodium pyrophosphate; $\mathrm{CuCl}_{2} ; \mathrm{LaCl}_{3} ; \mathrm{KCl} ; \mathrm{Al}$-humus complexes.

\section{INTRODUÇÃO}

Alumínio é o mais abundante elemento metálico da crosta terrestre e dos solos (Soon, 1993). A toxidez de suas formas lábeis às plantas cultivadas é bem conhecida e, em casos extremos, extensiva para algumas vegetações naturais. Sob determinadas circunstâncias, pode ser tóxico aos peixes, a outros organismos aquáticos e, mesmo, ao homem, tornandoo de considerável importância ambiental (Bache, 1986). Nos solos, além de estar presente em grandes teores em muitos minerais primários e na maioria dos minerais de argila e na gibbsita, frequentemente há importantes teores do elemento em estádios bem menos definidos (Lin \& Coleman, 1960), em diversas formas (Soon, 1993). Em consequência, sua química é complexa (Lin \& Coleman, 1960; Jarvis, 1986; Soon, 1993).

Em adição aos minerais aluminossilicados cristalinos e gibbsita, na fase sólida do solo o Al pode ocorrer como: (a) íon trocável; (b) adsorvido ou precipitado como compostos de Al-hidróxi (revestimentos de superfície ou Al entre camada); (c) minerais secundários e outros como gibbsita, sulfatos (alunita, basalalunita, jurbanita) e fosfatos (variscita) de Al e aluminossilicatos amorfos, como alofana e imogolita; e (d) em complexos organometálicos (Bache, 1986). Essa última é a forma que mais influencia nas propriedades, organização e evolução dos solos ácidos (Urrutia et al., 1995). Entre esses, destacam-se os Espodossolos. 
$\mathrm{O} \mathrm{Al}$ associado a complexos como a matéria orgânica do solo (MOS) comumente é determinado por técnicas de dissolução seletiva (García-Rodeja et al., 2004). Um extrator comum para essa forma de $\mathrm{Al}$ (e $\mathrm{Fe}$ ) é o pirofosfato de sódio $0,1 \mathrm{~mol} \mathrm{~L}^{-1}$ (Kaiser \& Zech, 1996). A interpretação do extrato pirofosfato tem sido tema de debates contínuos desde que Jeanroy \& Guillet (1981) e Higashi et al. (1981) demonstraram que tanto formas orgânicas quanto inorgânicas de Fe de baixo grau de cristalinidade podem ser simultaneamente obtidas pelo extrator (Paterson et al., 1993). Em trabalho mais recente, Kaiser \& Zech (1996) afirmaram que o extrator pirofosfato não deveria mais ser utilizado para estimar metais em complexos húmicos. Segundo eles, tanto o conteúdo de Fe quanto o de $\mathrm{Al}$ associados à MOS podem ser superestimados, enfatizando que o $\mathrm{Al}$ extraído com pirofosfato não pode ser atribuído unicamente à associação do elemento à fração húmica do solo, mas também à dissolução alcalina de gibbsita e à peptização de hidróxidos de $\mathrm{Al}$ associados à matéria orgânica neles adsorvida. Em vista da comprovada falta de seletividade do extrator pirofosfato para determinados solos e horizontes, temse sugerido que, para a adequada caracterização do $\mathrm{Al}$ complexado à matéria orgânica, extratores não tamponados de cloro, como $\mathrm{CuCl}_{2}$ (Juo \& Kamprath, 1979; Hargrove \& Thomas, 1981) e $\mathrm{LaCl}_{3}$ (Bloom et al., 1979b), podem dar melhores resultados (Oates \& Kamprath, 1983b).

Juo \& Kamprath (1979) foram os primeiros a propor $\mathrm{CuCl}_{2} \quad 0,5 \mathrm{~mol} \mathrm{~L}^{-1}$ como extrator de $\mathrm{Al}$ potencialmente reativo nos solos ácidos. No entanto, a interpretação dos resultados obtidos em solos minerais com respeito ao $\mathrm{Al}$ associado à matéria orgânica é dificultada, uma vez que $\mathrm{CuCl}_{2}$ pode, pelo menos parcialmente, extrair Al-hidróxi entre camadas e da superfície dos minerais de argila (Hargrove \& Thomas, 1984), presumivelmente devido à despolimerização induzida pelo baixo $\mathrm{pH}$ do extrator (Juo \& Kamprath, 1979; Soon, 1993).

Bloom et al. (1979b) propuseram o uso de $\mathrm{LaCl}_{3}$ $0,33 \mathrm{~mol} \mathrm{~L}^{-1}$ para a extração de $\mathrm{Al}$ associado à matéria orgânica dos solos. Comparando os dois extratores, Oates \& Kamprath (1983a) encontraram que $\mathrm{CuCl}_{2}$ $0,5 \mathrm{~mol} \mathrm{~L}^{-1}$ foi mais efetivo na remoção de $\mathrm{Al}$ dos complexos orgânicos que $\mathrm{LaCl}_{3} 0,33 \mathrm{~mol} \mathrm{~L}^{-1}$. Este último extrator possivelmente remove um conteúdo de $\mathrm{Al}$ que está relacionado à acidez titulável e, por isso, ao requerimento de calcário dos solos ácidos, tal como constatado por Hargrove \& Thomas (1984). Esses autores complementam que seu uso para tal fim parece promissor, mas necessita de ampla avaliação.

Outra fração de interesse aos estudos de fracionamento do $\mathrm{Al}$ nos solos por meio de técnicas de dissoluções seletivas é aquela extraída com $\mathrm{KCl}$ $1 \mathrm{~mol} \mathrm{~L}^{-1}$ (García-Rodeja et al., 2004), geralmente referida como Al trocável (Lin \& Coleman, 1960; Oates \& Kamprath, 1983a,b; Gillman \& Sumpter, 1985). Além de ser o método-padrão para formas de $\mathrm{Al}$ trocável (Dahlgren \& Walker, 1994), é utilizado como critério tanto para predizer o requerimento de calcário em solos ácidos (Oates \& Kamprath, 1983a,b; Hargrove \& Thomas, 1984) quanto em alguns sistemas de classificação de solos (Gillman \& Sumpter, 1985).

Extratores não tamponados de $\mathrm{Cl}$ possuem a habilidade de remover o $\mathrm{Al}$ que se encontra unido à matéria orgânica em diferentes graus de estabilidade, colaborando para o entendimento da química dos complexos Al-húmus (García-Rodeja et al., 2004). Tais complexos têm forte influência no tamponamento do pH e regulação da atividade do $\mathrm{Al}$ (Bloom et al., 1979a,b; Takahashi et al., 1995), além de terem importante papel na gênese da maioria dos solos estudados. Associado a isso, as recentes propostas de utilizá-los $\left(\mathrm{CuCl}_{2} \mathrm{e} \mathrm{LaCl}_{3}\right)$ como métodos alternativos do $\mathrm{Al}$ complexado à matéria orgânica têm despertado interesse nos estudos que avaliam a gênese dos Espodossolos, como o de Sauer et al. (2007), com potencial para incorporá-los aos critérios diagnósticos químicos na definição de horizontes espódicos e na classificação desses solos nos sistemas taxonômicos. Neste estudo, objetivou-se avaliar a eficácia dos reagentes não tamponados de $\mathrm{Cl}, \mathrm{KCl}, \mathrm{LaCl}_{3} \mathrm{e} \mathrm{CuCl}_{2}$, na dissolução do $\mathrm{Al}$, sobretudo daquele ligado à matéria orgânica em Espodossolos e Neossolos Quartzarênicos representativos do litoral paulista, comparando-os ao pirofosfato.

\section{MATERIAL E MÉTODOS}

\section{Meio físico}

Foram coletadas amostras de 31 perfis de solos (27 Espodossolos e quatro Neossolos Quartzarênicos) descritos sob vegetação de restinga em três municípios do Estado de São Paulo: Bertioga, Cananeia e Ilha Comprida.

O clima do litoral do Estado de São Paulo é do tipo "Af", tropical úmido ou superúmido, com chuvas distribuídas durante todo o ano, conforme classificação climática de Köppen (Setzer, 1966). Segundo dados climatológicos da Estação Meteorológica do DAEE em Bertioga ( $24^{\circ} 45,6^{\prime}$ 'S e $\left.46^{\circ} 04,2^{\prime} \mathrm{W}\right)$, obtidos entre 1941 e 1970, a temperatura e precipitação pluvial médias anuais são, respectivamente, $24,8^{\circ} \mathrm{C}$ e $3.200 \mathrm{~mm}$, sendo considerada uma das regiões mais úmidas do Brasil (Martins et al., 2008).

Os perfis estudados desenvolveram-se nos sedimentos arenosos quaternários marinhos (Suguio \& Martin, 1978; Suguio et al., 1999). Os solos sob vegetação de restinga englobam aqueles classificados como Espodossolos e Neossolos Quartzarênicos (Oliveira et al., 1992).

\section{Trabalhos de campo}

Após a seleção dos locais de amostragem, foram abertas trincheiras e descritos e amostrados os perfis, 
conforme Santos et al. (2005), sendo os solos classificados segundo a Embrapa (2006). A descrição e localização detalhadas dos solos e paisagens estudadas são encontradas em Coelho et al. (2010a).

\section{Análises laboratoriais}

No laboratório, as amostras de solo foram secas ao ar, destorroadas com um martelo de borracha, quando necessário, e passadas em peneira com malha de $2 \mathrm{~mm}$, obtendo-se a fração terra fina seca ao ar, em que foram realizadas as análises químicas. $\mathrm{O} \mathrm{pH}$ foi determinado tanto em água, utilizando relação solo:solução 1:2,5 após agitação e repouso de $1 \mathrm{~h}$, quanto na solução de equilíbrio, após a extração com $\mathrm{CuCl}_{2}(\mathrm{pH} \mathrm{CuCl})_{2}, \mathrm{LaCl}_{3}(\mathrm{pH} \mathrm{LaCl})_{3}$ e $\mathrm{KCl}(\mathrm{pH} \mathrm{KCl})$ e agitação, centrifugação e filtragem do sobrenadante. O C orgânico (Corg) e a capacidade de troca de cátions (CTC) foram obtidos segundo a Embrapa (1997). Essa última corresponde à soma dos cátions $\mathrm{Ca}^{2+}, \mathrm{Mg}^{2+}, \mathrm{K}^{+}$

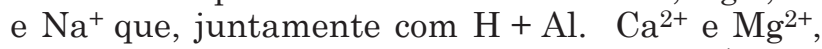
foram extraídos com solução de $\mathrm{KCl} 1 \mathrm{~mol} \mathrm{~L}^{-1} ; \mathrm{K}^{+} \mathrm{e}$ $\mathrm{Na}^{+} \mathrm{H}_{2} \mathrm{SO}_{4} 0,0125 \mathrm{~mol} \mathrm{~L}^{-1}+\mathrm{HCl} 0,05 \mathrm{~mol} \mathrm{~L}^{-1} ; \mathrm{H}+\mathrm{Al}$ com acetato de cálcio $0,5 \mathrm{~mol} \mathrm{~L}^{-1} \mathrm{a} \mathrm{pH} \mathrm{7,0.} \mathrm{Os} \mathrm{teores}$ de $\mathrm{Ca}^{2+} \mathrm{e} \mathrm{Mg}^{2+}$ foram determinados por espectroscopia de absorção atômica, $\mathrm{K}^{+}$e $\mathrm{Na}^{+}$por fotometria de chama $\mathrm{e} \mathrm{H}+\mathrm{Al}$ por titulometria.

Os seguintes procedimentos foram utilizados para a extração do Al: (a) Extração com oxalato ácido de amônio de acordo com Buurman et al. (1996): relação solo:solução de 1:50, agitando por $4 \mathrm{~h}$, no escuro. A suspensão foi centrifugada por 15 min a $2.500 \mathrm{rpm}$ após a adição de quatro gotas de "superfloc". O sobrenadante foi filtrado através de papel- filtro lavado em ácido (7-11 $\mu \mathrm{m}$ de diâmetro de poro); (b) Extração com pirofosfato de sódio $0,1 \mathrm{~mol} \mathrm{~L}^{-1}\left(\mathrm{pH} 10 ; \mathrm{Al}_{\mathrm{p}}\right.$ ): relação solo:solução 1:100, agitando por $16 \mathrm{~h}$ (Buurman et al., 1996); (c) Extração com $\mathrm{CuCl}_{2}$ $0,5 \mathrm{~mol} \mathrm{~L}^{-1}\left(\mathrm{pH} 3 ; \mathrm{Al}_{\mathrm{Cu}}\right): 5 \mathrm{~g}$ de solo foram agitados durante 5 min com $50 \mathrm{~mL}$ da solução extratora. Após a agitação, a suspensão permaneceu em repouso durante $12 \mathrm{~h}$, seguida de nova agitação durante $30 \mathrm{~min}$ e filtragem através de papel-filtro lavado em ácido. Lavou-se o solo contido no papel-filtro com $\mathrm{CuCl}_{2}$ até completar o volume para $100 \mathrm{~mL}$ (Juo \& Kamprath, 1979); (d) Extração com $\mathrm{LaCl}_{3} 0,33 \mathrm{~mol} \mathrm{~L}^{-1}$ (pH 4; $\mathrm{Al}_{\mathrm{La}}$ ): agitaram-se $10 \mathrm{~g}$ de solo durante $2 \mathrm{~h}$ com $50 \mathrm{~mL}$ da solução extratora. A suspensão foi filtrada utilizando-se papel-filtro lavado em ácido, e o solo nele retido foi lavado com $\mathrm{LaCl}_{3}$ até completar o volume para $100 \mathrm{~mL}$ (Hargrove \& Thomas, 1981); (e) Extração com $\mathrm{KCl} 1 \mathrm{~mol} \mathrm{~L}^{-1}\left(\mathrm{pH} 5 ; \mathrm{Al}_{\mathrm{K}}\right)$ : $5 \mathrm{~g}$ de solo foram agitados durante 5 min com $50 \mathrm{~mL}$ da solução extratora. Após a agitação, procedeu-se imediatamente à filtragem da suspensão em papel-filtro lavado em ácido (Raij et al., 2001).

$\mathrm{O} \mathrm{Al}$ nos extratos foi determinado por espectroscopia de absorção atômica. Os resultados apresentados para esse elemento representam a média das análises realizadas em duplicata, as quais diferiram entre si em menos de $10 \%$.

\section{RESULTADOS E DISCUSSÃO}

A habilidade dos diferentes extratores em remover $\mathrm{Al}$ unido à matéria orgânica dos horizontes e solos estudados (Figura 1) segue, na maioria das amostras, aquela já descrita por vários autores (Bloom et al., 1979b; Hargrove \& Thomas, 1981; Oates \& Kamprath, 1983a; Hargrove \& Thomas, 1984; Urrutia et al., 1995; García-Rodeja et al., 2004): $\mathrm{Al}_{\mathrm{p}}>\mathrm{Al}_{\mathrm{Cu}}>\mathrm{Al}_{\mathrm{La}}>$ $\mathrm{Al}_{\mathrm{K}}$. No entanto, algumas amostras não seguiram essa tendência, o que será discutido nos itens subsequentes. Pelo exame da figura 1, observa-se que os horizontes dotados de subscrito "s" (Bs, Bhs e Bsm) detêm os maiores conteúdos médios de $\mathrm{Al}_{\mathrm{p}}$, enquanto $\mathrm{Al}_{\mathrm{Cu}}$ e $\mathrm{Al}_{\mathrm{La}}$ foram mais eficientemente removidos dos horizontes Bhm.

\section{Estabilidade da interação Al-húmus}

García-Rodeja et al. (2004) estabeleceram os seguintes parâmetros para avaliar o grau de estabilidade da interação do $\mathrm{Al}$ com a MOS, expressos em ordem decrescente da estabilidade: $\mathrm{Al}_{\mathrm{p}}-\mathrm{Al}_{\mathrm{Cu}}>\mathrm{Al}_{\mathrm{Cu}}{ }^{-}$ $\mathrm{Al}_{\mathrm{La}}>\mathrm{Al}_{\mathrm{La}}-\mathrm{Al}_{\mathrm{K}}$. Baseado nessas relações, observou-se que os menores valores da diferença entre as diferentes formas de $\mathrm{Al}$ correspondem àquelas mais lábeis da interação $\mathrm{Al}$-húmus $\left(\mathrm{Al}_{\mathrm{La}}-\mathrm{Al}_{\mathrm{K}}\right)$, as quais são, em geral, encontradas nos horizontes $\mathrm{A}$ e $\mathrm{C}$, enquanto os maiores valores correspondem às formas mais estáveis da interação $\left(\mathrm{Al}_{\mathrm{p}}-\mathrm{Al}_{\mathrm{Cu}}\right)$, nos horizontes Bhs e Bsm (Figura 2). Assim, a seguinte sequência de estabilidade da interação Al-húmus foi observada nos solos estudados: $\mathrm{A}<\mathrm{C}<\mathrm{Bh}<\mathrm{Bhm}<\mathrm{Bs}<\mathrm{Bsm}<\mathrm{Bhs}$. O máximo valor, 744,11 $\mathrm{mmol}_{\mathrm{c}} \mathrm{kg}^{-1}$, foi registrado na diferença $\mathrm{Al}_{\mathrm{p}}-\mathrm{Al}_{\mathrm{Cu}}$ de um horizonte $\mathrm{Bs}$ descrito em um perfil bem drenado (perfil P5, horizonte Bs6), cujos

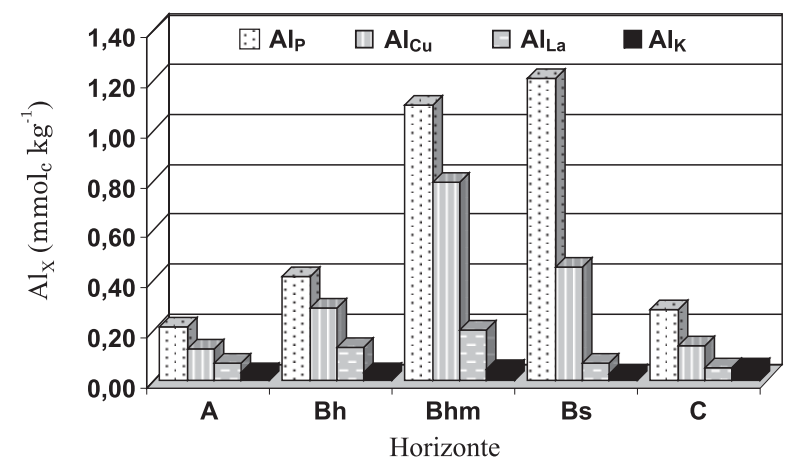

Figura 1. Conteúdo médio de Al extraído pelos reagentes pirofosfato de sódio $\left(\mathrm{Al}_{\mathrm{p}}\right), \mathrm{CuCl}_{2}\left(\mathrm{Al}_{\mathrm{Cu}}\right)$, $\mathrm{LaCl}_{3}\left(\mathrm{Al}_{\mathrm{La}}\right)$ e $\mathrm{KCl}\left(\mathrm{Al}_{\mathrm{K}}\right)$ em todos os horizontes e perfis estudados. $\mathrm{Al}_{\mathrm{x}}$ simboliza as diferentes formas de Al. Horizontes A $(n=16)$ incluem aqueles intermediários $\mathrm{AB}, \mathrm{AE}$ e AC. Horizontes Bs $(n=48)$ compreendem aqueles descritos como Bs, Bhs e Bsm. Horizontes $\mathrm{C}(\mathrm{n}=36)$ incluem aqueles intermediários CA e CB. Horizontes Bh e Bhm com número de amostras (n) de 41 e 13, respectivamente. Média dos valores diferentes de zero. 


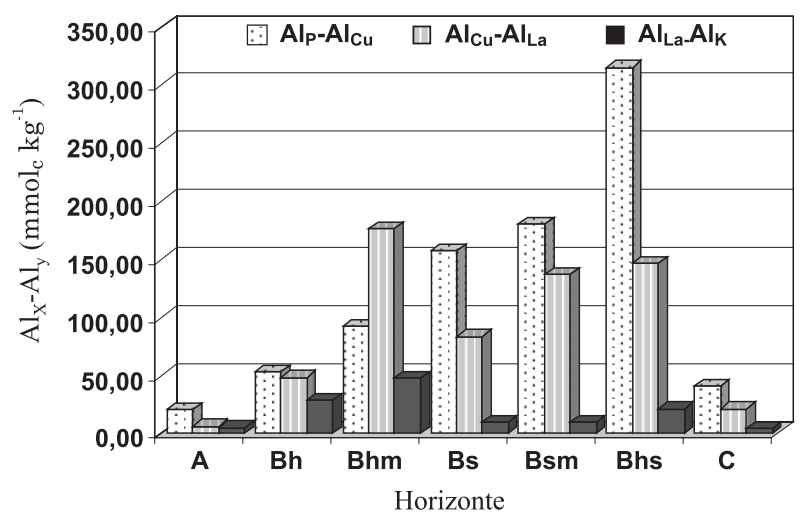

Figura 2. Conteúdo médio das diferentes formas de Al nos horizontes estudados e que expressam a seguinte ordem decrescente de estabilidade da interação Al-húmus: $\mathrm{Al}_{\mathrm{p}}-\mathrm{Al}_{\mathrm{Cu}} ; \mathrm{Al}_{\mathrm{Cu}}-\mathrm{Al}_{\mathrm{La}} ; \mathrm{Al}_{\mathrm{La}}-\mathrm{Al}_{\mathrm{K}}$. $\mathrm{Al}_{\mathrm{x}}-\mathrm{Al}_{\mathrm{y}}$ simboliza as diferentes formas de $\mathrm{Al}$. Horizontes A incluem aqueles intermediários $\mathrm{AB}, \mathrm{AE}$ e AC. Horizontes $\mathrm{C}$ incluem aqueles intermediários CA e CB. Média dos valores positivos e diferentes de zero.

dados químicos convergem para a presença de formas inorgânicas de $\mathrm{Al}\left(\mathrm{Al}_{\mathrm{p}} / \mathrm{Al}_{\mathrm{o}}=0,65 ;\left(\mathrm{Al}_{\mathrm{p}} /\right.\right.$ Corg $) 100=$ 45,27; Coelho et al., 2010b) e, portanto, há uma provável ação do pirofosfato sobre tais formas. Outros autores também relataram a maior estabilidade dos complexos Al-húmus em horizontes Bs comparativamente aos Bh (Urrutia et al., 1995). Os valores de pH superiores a 4,5 em muitos desses horizontes com subscrito "s" (valores médio, mínimo e máximo em todos os 48 horizontes estudados de, respectivamente, 4,$7 ; 3,2$; e 6,0) são parcialmente responsáveis por esses resultados. Segundo Ponette et al. (1996), acima desse valor espécies de hidróxi-Al predominam e tendem a se polimerizar, formando complexos mais estáveis com a MOS.

\section{Al extraído com $\mathrm{CuCl}_{2}\left(\mathrm{Al}_{\mathrm{Cu}}\right)$}

A porcentagem de $\mathrm{Al}$ extraído pelo $\mathrm{CuCl}_{2}$ em relação ao pirofosfato variou de 17,8 a 125,8 \%, com média de
$63,6 \%(n=180)$. Apenas duas amostras tiveram seus valores de $\mathrm{Al}$ no extrato $\mathrm{CuCl}_{2}$ superior ao do pirofosfato (Figura 3). Essas se referem aos horizontes BCg e 2Cg de um mesmo perfil (perfil P8; Quadro 1), os quais se encontram permanentemente submersos e com baixos conteúdos de C orgânico. No entanto, as diferenças entre esses extratores são pequenas (5,12 $\mathrm{mmol}_{\mathrm{c}} \mathrm{kg}^{-1}$ no horizonte $\mathrm{BCg}$ e 17,60 $\mathrm{mmol}_{\mathrm{c}} \mathrm{kg}^{-1}$ no horizonte $2 \mathrm{Cg}$ ), o que sugere similar capacidade de extração em ambos os métodos, possivelmente com ligeira contribuição da fase mineral no $\mathrm{Al}_{\mathrm{Cu}}$, fato também constatado e assim interpretado por GarcíaRodeja et al. (2004) em alguns horizontes C e superficiais de solos vulcânicos europeus, com baixos conteúdos de Corg.

Dahlgren \& Walker (1993) relataram valores de $\mathrm{Al}_{\mathrm{Cu}}$ em relação ao $\mathrm{Al}_{\mathrm{p}}$ variando de 50 a $80 \%$ em

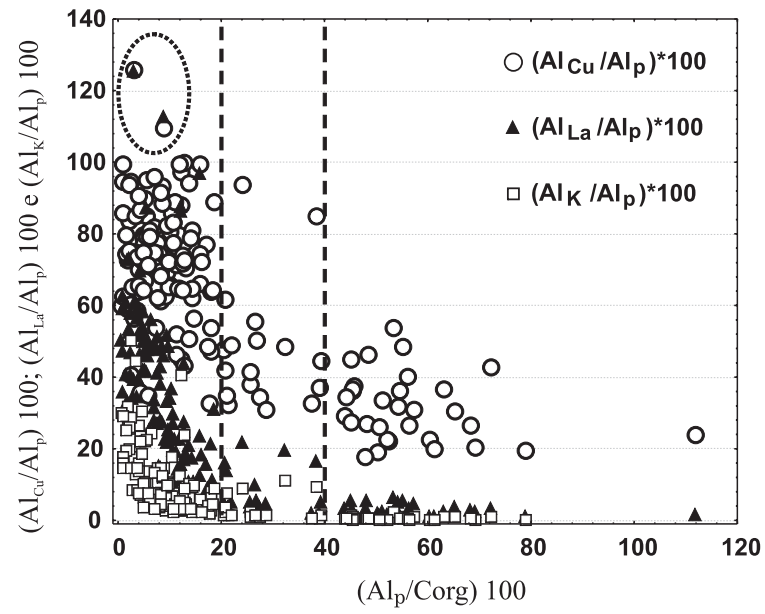

Figura 3. Relação entre a saturação por Al extraído por pirofosfato no húmus dos solos estudados ( $\left.\left(\mathrm{Al}_{\mathrm{p}} / \mathrm{Corg}\right) 100\right)$ e a percentagem de $\mathrm{Al}$ removido por $\mathrm{CuCl}_{2}, \mathrm{LaCl}_{3}$ e $\mathrm{KCl}$ em relação ao elemento no extrato pirofosfato $\left(\left(\mathrm{Al}_{\mathrm{Cu}} / \mathrm{Al}_{\mathrm{p}}\right) 100\right) ;\left(\mathrm{Al}_{\mathrm{La}} / \mathrm{Al}_{\mathrm{p}}\right)$ 100 e $\left.\left(\mathrm{Al}_{\mathrm{K}} / \mathrm{Al}_{\mathrm{p}}\right) \mathrm{100}\right)$, respectivamente). Em duas das amostras (circundadas), $\mathrm{CuCl}_{2}$ e $\mathrm{LaCl}_{3}$ excederam a capacidade do pirofosfato em remover $\mathrm{Al}$ orgânico.

Quadro 1. Valores de Corg, de pH, da relação $\mathrm{Al}_{\mathrm{p}} / \mathrm{Al}_{\mathrm{o}}$ e de $\mathrm{Al}$ extraído com $\mathrm{LaCl}_{3}$, sua eficácia em relação ao pirofosfato $\left(\left(\mathrm{Al}_{\mathrm{La}} / \mathrm{Al} \mathrm{p}_{\mathrm{p}}\right) 100\right)$ e ao $\mathrm{CuCl}_{2}\left(\left(\mathrm{Al}_{\mathrm{La}} / \mathrm{Al}_{\mathrm{Cu}}\right) 100\right)$ de perfis selecionados

\begin{tabular}{|c|c|c|c|c|c|c|c|}
\hline Horizonte & Profundidade $^{(1)}$ & Corg & $\mathbf{p H}$ & $\mathrm{Al}_{\mathrm{p}} / \mathrm{Al}_{\mathrm{o}}$ & $\mathrm{Al}_{\mathrm{La}}$ & $\left(\mathrm{Al}_{\mathrm{La}} / \mathrm{Al}_{\mathrm{p}}\right) 100$ & $\left(\mathrm{Al}_{\mathrm{La}} / \mathrm{Al}_{\mathrm{Cu}}\right) 100$ \\
\hline & $\mathrm{cm}$ & $\mathrm{g} \mathrm{kg}^{-1}$ & & & $\mathrm{mmol}_{\mathrm{c}} \mathrm{kg}^{-1}$ & $\%$ & - \\
\hline $\mathrm{A} 1$ & \multicolumn{7}{|c|}{ Perfil P1 - Neossolo Quartzarênico órtico típico, A moderado, excessivamente drenado } \\
\hline $\begin{array}{l}\text { A1 } \\
\text { A2 }\end{array}$ & $8-25$ & $\begin{array}{l}19,83 \\
17,57\end{array}$ & $\begin{array}{l}4,2 \\
4,0\end{array}$ & $\begin{array}{l}1,49 \\
1,23\end{array}$ & $\begin{array}{l}11,61 \\
23,55\end{array}$ & $\begin{array}{l}35,35 \\
39,26\end{array}$ & $\begin{array}{l}56,24 \\
69,63\end{array}$ \\
\hline $\mathrm{AC}$ & $25-38$ & 9,69 & 4,5 & 0,99 & 20,33 & 15,64 & 34,74 \\
\hline $\mathrm{C} 1$ & $38-84$ & 8,18 & 4,8 & 1,07 & 13,11 & 3,29 & 11,23 \\
\hline $\mathrm{C} 2$ & $84-140$ & 3,94 & 4,9 & 0,77 & 11,78 & 4,98 & 15,49 \\
\hline C3 & $140-160$ & 3,63 & 4,9 & 0,69 & 10,67 & 4,60 & 14,90 \\
\hline $\mathrm{C} 4$ & $160-175$ & 3,33 & 4,7 & 0,56 & 9,22 & 3,82 & 12,49 \\
\hline C5 & $175-210$ & 2,00 & 4,8 & 0,49 & 5,29 & 5,77 & 11,83 \\
\hline Plácico & 180 & 9,39 & 4,2 & 0,63 & 18,33 & 2,54 & 12,50 \\
\hline
\end{tabular}


Quadro 1. Continuação

\begin{tabular}{|c|c|c|c|c|c|c|c|}
\hline Horizonte & Profundidade $^{(1)}$ & Corg & pH & $\mathrm{Al}_{\mathrm{p}} / \mathrm{Al}_{\mathrm{o}}$ & $\mathrm{Al}_{\mathrm{La}}$ & $\left(\mathrm{Al}_{\mathrm{La}} / \mathrm{Al}_{\mathrm{p}}\right) 100$ & $\left(\mathrm{Al}_{\mathrm{La}} / \mathrm{Al}_{\mathrm{Cu}}\right) 100$ \\
\hline & $\mathrm{cm}$ & $\mathrm{g} \mathrm{kg}^{-1}$ & & & $\mathrm{mmol}_{\mathrm{c}} \mathrm{kg}^{-1}$ & \multicolumn{2}{|c|}{$\bar{\square} \%$} \\
\hline \multirow{2}{*}{\multicolumn{8}{|c|}{ 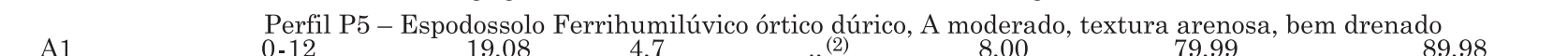 }} \\
\hline $\begin{array}{l}\mathrm{A} 1 \\
\mathrm{~A} 2\end{array}$ & & & & & $\begin{array}{r}8,00 \\
1455\end{array}$ & & $\begin{array}{l}89,98 \\
99,91\end{array}$ \\
\hline $\mathrm{A} 2$ & & 11,21 & 4,3 & .. & 14,55 & \multirow{2}{*}{72,77} & 99,91 \\
\hline $\mathrm{E}$ & $27-57$ & 0,63 & 4,5 & .. & 1,11 & & \multirow{2}{*}{$\begin{array}{l}89,99 \\
64,45\end{array}$} \\
\hline Bhg & $57-75$ & 10,70 & 4,0 & 1,09 & 48,22 & \multirow{2}{*}{\multicolumn{2}{|c|}{$\begin{array}{l}45,92 \\
22,55\end{array}$}} \\
\hline Bh1 & $52-75$ & 31,49 & 4,0 & 0,91 & 71,77 & & \\
\hline Bh2 & $63-80$ & 33,37 & 3,9 & 1,15 & 105,10 & \multicolumn{2}{|r|}{32,42} \\
\hline Bh3 & $55-95$ & 26,44 & 4,2 & 1,14 & 37,00 & \multicolumn{2}{|r|}{11,08} \\
\hline Bh4 4 & $126-150$ & 32,11 & 4,4 & 0,78 & 35,55 & \multicolumn{2}{|r|}{9,41} \\
\hline Bh5 & $73-112$ & 27,39 & 4,2 & 0,99 & 44,22 & \multicolumn{2}{|r|}{10,82} \\
\hline Bs1 & $78-101$ & 12,59 & 4,7 & 0,66 & 17,00 & \multicolumn{2}{|r|}{9,70} \\
\hline Bs2 & $82-85$ & 5,35 & 4,8 & 0,56 & 11,33 & \multicolumn{2}{|r|}{10,55} \\
\hline Bs3 & $95-126$ & 10,07 & 4,6 & 0,65 & 12,67 & 2,25 & 11,71 \\
\hline Bs4 & $85-180$ & 16,37 & 4,4 & 0,86 & 37,44 & 5,24 & 11,75 \\
\hline Bs5 & $130-150$ & 3,15 & 4,8 & 0,42 & 5,67 & 2,58 & 7,02 \\
\hline Bs6 & $54-120$ & 23,30 & 4,5 & 0,65 & 34,66 & 2,96 & 8,11 \\
\hline Bs7 & $155-180$ & 13,85 & 4,7 & 0,68 & 13,44 & 1,67 & 7,45 \\
\hline $\mathrm{C}$ & $54-112$ & 1,57 & 5,1 & 0,36 & 6,11 & 6,55 & 12,13 \\
\hline Plácico & & & & & & & \\
\hline Hacter & $\begin{array}{l}\text { Perfil P8 - Espodo } \\
0-13\end{array}$ & o Ferrih & $\begin{array}{l}\text { vico h } \\
3.7\end{array}$ & órfico esp & $\underset{24.44}{\text { rênico, } \mathrm{m}}$ & $\begin{array}{l}\text { ado, textura aren } \\
50,57\end{array}$ & a, mal drenado \\
\hline $\mathrm{A} 2$ & $13-23$ & 12,28 & 3,9 &.. & 5,11 & 47,17 & 50,48 \\
\hline EA1 & $17-29$ & 5,67 & 4,3 &.. & 2,76 & .. & 61,99 \\
\hline EA2 & $29-36$ & 5,04 & 4,6 & .. & 3,57 & & 60,18 \\
\hline Bh1 & $36-42$ & 17,31 & 4,2 & 1,01 & 55,44 & 58,36 & 75,60 \\
\hline $\mathrm{Bh} 2$ & $42-82$ & 21,41 & 3,6 & 1,20 & 63,55 & 45,94 & 63,55 \\
\hline Bhs & $53-93$ & 10,39 & 3,4 & 0,98 & 43,77 & 36,99 & 42,93 \\
\hline $\mathrm{Bs}$ & $93-114$ & 4,41 & 3,6 & 0,98 & 26,89 & 43,60 & 56,13 \\
\hline $\mathrm{BCg}$ & $114-129$ & 5,35 & 3,1 & 0,99 & 58,22 & 112,68 & 102,51 \\
\hline Cg1 & $129-150$ & 10,07 & 3,4 & 0,73 & 52,33 & 87,21 & 91,74 \\
\hline $2 \mathrm{Cg} 2$ & $150-170$ & 21,72 & 3,3 & 0,68 & 85,77 & 125,52 & 99,82 \\
\hline & Perfil P10 - Espo & solo Hun & co hid & fico espes & nico, A moc & o, textura arenos & mal drenado \\
\hline A & $0-7$ & 67,37 & 3,9 & 0,61 & 24,55 & 113,31 & 151,34 \\
\hline $\mathrm{AE}$ & $7-11$ & 20,46 & 4,1 & .. (2) & 6,78 & .. & 83,17 \\
\hline EA & $11-30$ & 8,81 & 4,2 &.. & 3,94 & .. & 69,45 \\
\hline $\mathrm{E}$ & $18-48$ & 1,26 & 4,9 & .. & 1,00 & .. & \\
\hline EB & $35-74$ & 1,57 & 4,7 & & 2,63 & & 88,87 \\
\hline $\mathrm{BE}$ & $60-83$ & 4,09 & 4,5 & $0, \ddot{51}$ & 8,67 & 61,17 & 81,62 \\
\hline Bh1 & $83-103$ & 15,11 & 3,9 & 3,14 & 39,22 & 53,48 & 73,53 \\
\hline $\mathrm{Bh} 2$ & $90-117$ & 19,52 & 4,1 & 1,58 & 76,44 & 55,93 & 79,79 \\
\hline $\mathrm{Bhm}$ & $117-150$ & 18,57 & 4,1 & 1,64 & 63,44 & 41,37 & 56,97 \\
\hline & Perfil P28 - Esp & solo Fer & iilúvic & o espessa & co, A fraco, & ura arenosa, bem & enado \\
\hline A & $0-21$ & 5,71 & 4,1 & .. & 1,67 & .. & 74,99 \\
\hline $\mathrm{E}$ & $21-126$ & 0,93 & 5,0 & & & .. & \\
\hline Bhs1 & $54-73$ & 11,27 & 4,6 & 1,10 & 16,78 & 4,68 & 15,00 \\
\hline Bhs2 & $65-88$ & 10,50 & 5,0 & 1,27 & 22,00 & 3,35 & 12,53 \\
\hline Bhs3 & $60-108$ & 13,89 & 4,8 & 1,47 & 40,44 & 5,44 & 20,15 \\
\hline Bhs4 & $77-177$ & 10,81 & 5,1 & 0,87 & 9,44 & 1,51 & 6,69 \\
\hline Bhs5 & $111-200$ & 6,33 & 5,1 & 0,67 & 7,55 & 2,22 & 4,80 \\
\hline Bhs6 & $134-160$ & 8,49 & 4,9 & 0,62 & 8,11 & 1,90 & 5,19 \\
\hline Bhs7 & $150-200$ & 6,79 & 5,0 & 0,68 & 6,33 & 1,66 & 6,27 \\
\hline & Perfil P29 - Esp & solo Hur & co órt & rico, $\mathrm{A}$ fro & cextura are & imperfeitamente & renado \\
\hline A & $0-14$ & 64,52 & $\ldots{ }^{(3)}$ & .. & 9,33 & .. & 72,69 \\
\hline $\mathrm{E}$ & $14-60$ & 2,16 & 4,9 & & & .. & \\
\hline $\mathrm{Bh}$ & $47-70$ & 36,12 & 3,8 & $1,2 \ddot{2}$ & 81,77 & $56,7 \ddot{2}$ & 77,20 \\
\hline Bhm1 & $60-87$ & 50,94 & 3,7 & 1,06 & 114,88 & 52,22 & 57,50 \\
\hline $\mathrm{Bhm} 2$ & 87-104 & 18,52 & 4,4 & 1,06 & 14,67 & 5,75 & 8,92 \\
\hline Bs & $104-115$ & 9,11 & 4,6 & 1,05 & 11,00 & 5,00 & 10,15 \\
\hline & Perfil P31 - Espod & lo Ferril & úvico & dúrico, $\mathrm{A}$ & o, textura a & sa, imperfeitame & drenado \\
\hline A & $0-10$ & 22,85 & 4,7 & .. & 4,00 & .. & 50,62 \\
\hline $\mathrm{E}$ & $10-18$ & 2,01 & 4,9 & & & .. & \\
\hline $\mathrm{Bh}$ & $18-40$ & 28,40 & 3,8 & 1,21 & 86,44 & 47,93 & 66,93 \\
\hline $\mathrm{Bhm}$ & $33-44$ & 33,34 & 3,8 & 1,11 & 117,99 & 29,74 & 35,61 \\
\hline Bs1 & $40-76$ & 5,56 & 4,5 & 0,87 & 4,67 & 1,71 & 4,96 \\
\hline Bs2 & $58-105$ & 7,56 & 4,5 & 0,46 & 21,78 & 9,82 & 17,67 \\
\hline
\end{tabular}

(1) Os valores iniciais e finais das profundidades podem estar relacionados a transições entre horizontes do tipo descontínua; daí a sobreposição dos valores entre alguns horizontes. ${ }^{(2)}$ Não se aplica dado numérico. ${ }^{(3)}$ Dado numérico não-disponível. 
horizontes espódicos, o que difere consideravelmente daqueles aqui encontrados, os quais variaram entre 19 e $98 \%$.

Em vista do exposto, o pirofosfato extraiu mais $\mathrm{Al}$ que o $\mathrm{CuCl}_{2}$ na grande maioria das amostras, com diferenças entre 0,03 e 744,11 $\mathrm{mmol}_{\mathrm{c}} \mathrm{kg}^{-1}$ (Figura 1). A figura 3 evidencia que há forte tendência de que quanto maior é a saturação por Al no húmus, expressa pela equação $\left(\mathrm{Al}_{\mathrm{p}} / \mathrm{Corg}\right)$ 100, maior é a diferença entre a capacidade extratora do pirofosfato em relação ao $\mathrm{CuCl}_{2}$ e demais extratores. Dois fatores podem ser corresponsáveis por esse resultado (Coelho et al., 2010c): (1) ação do pirofosfato sobre formas inorgânicas de Al; e, sobretudo, (2) maior estabilidade da interação Al-húmus com o aumento da saturação do elemento na MOS.

\section{$\mathrm{Al}$ extraído com $\mathrm{LaCl}_{3}\left(\mathrm{Al}_{\mathrm{La}}\right)$}

$\mathrm{O}$ conteúdo de $\mathrm{Al}$ extraído por $\mathrm{LaCl}_{3}$ variou entre 0,3 e $156 \mathrm{mmol}_{\mathrm{c}} \mathrm{kg}^{-1}$, não muito distante daqueles relatados por García-Rodeja et al. (2004) em solos vulcânicos europeus $\left(0,2\right.$ a $\left.190 \mathrm{mmol}_{\mathrm{c}} \mathrm{kg}^{-1}\right)$. Há tendência de seu aumento com o conteúdo de $\mathrm{C}$ orgânico. $\mathrm{O}$ quadro 2 mostra os coeficientes de correlação da curva de regressão linear entre os teores de Corg e de $\mathrm{Al}$ obtidos pelos diferentes extratores, considerando-se apenas os horizontes B espódicos. A menor dispersão dos dados se dá no $\mathrm{LaCl}_{3}\left(\mathrm{r}=0,88^{* * *}\right)$, possivelmente devido à ineficácia do extrator para formas inorgânicas de $\mathrm{Al}$ e à abundância do elemento em complexos de esfera externa para os horizontes espódicos estudados e, portanto, de menor estabilidade da interação Al-húmus (Urrutia et al., 1995).

A quantidade de $\mathrm{Al}$ extraído por $\mathrm{LaCl}_{3}$ decresceu com o aumento do $\mathrm{pH}$ medido em água e foi menor que $20 \mathrm{mmol}_{\mathrm{c}} \mathrm{kg}^{-1} \mathrm{em}$ valores de $\mathrm{pH}$ superiores a 5,0 (Figura 4), corroborando a maior estabilidade da interação Al-húmus para valores de $\mathrm{pH}$ acima de 4,5.

A curva da figura $4 \mathrm{a}$, que relaciona $\mathrm{pH}_{2} \mathrm{O} \times \mathrm{Al}_{\text {La }}$, ajustou-se melhor ( $\mathrm{r}=0,61$; curva polinomial) em relação àquela observada para o $\mathrm{CuCl}_{2}\left(\mathrm{r}=-0,31^{* * *}\right.$; Figura $4 \mathrm{~b}$ ). Esse fato, associado à menor dispersão dos dados quando se relaciona tanto o Corg (Quadro 2) quanto a CTC do solo (Quadro 3) ao Al removido por $\mathrm{LaCl}_{3}$, sugere que, entre os extratores utilizados, $\mathrm{Al}_{\mathrm{La}}$

Quadro 2. Coeficiente de correlação $\mathrm{r}$ e número de amostras n na relação linear entre o carbono orgânico (Corg) e as seguintes variáveis: $\mathrm{Al}_{\mathrm{p}}, \mathrm{Al}_{\mathrm{Cu}}$, $\mathrm{Al}_{\mathrm{La}}$ e $\mathrm{Al}_{\mathrm{K}}$. Amostras referentes aos horizontes espódicos. Valor de $\mathrm{p}<0,0001 \mathrm{em}$ todas as correlações

\begin{tabular}{|c|c|c|c|c|c|c|c|c|}
\hline \multirow{2}{*}{ Variável } & \multicolumn{2}{|c|}{$\mathrm{Al}_{\mathrm{p}}$} & \multicolumn{2}{|c|}{$\mathrm{Al}_{\mathrm{Cu}}$} & \multicolumn{2}{|c|}{$\mathrm{Al}_{\mathrm{La}}$} & \multicolumn{2}{|c|}{$\mathrm{Al}_{\mathrm{K}}$} \\
\hline & $\mathbf{r}$ & $\mathbf{n}$ & $\mathbf{r}$ & $\mathbf{n}$ & $\mathbf{r}$ & $\mathbf{n}$ & $\mathbf{r}$ & $\mathbf{n}$ \\
\hline Corg & 0,36 & 106 & 0,67 & 108 & 0,88 & 105 & 0,62 & 106 \\
\hline
\end{tabular}

é o que melhor se relaciona aos componentes responsáveis pelo desenvolvimento de cargas nos solos estudados. Portanto, é a fração mais lábil da interação

(a) $\mathrm{Al}_{\mathrm{La}}=10,16\left(\mathrm{pH} \mathrm{H}_{2} \mathrm{O}\right)^{2}-113,61\left(\mathrm{pH} \mathrm{H}_{2} \mathrm{O}\right)+320,96$

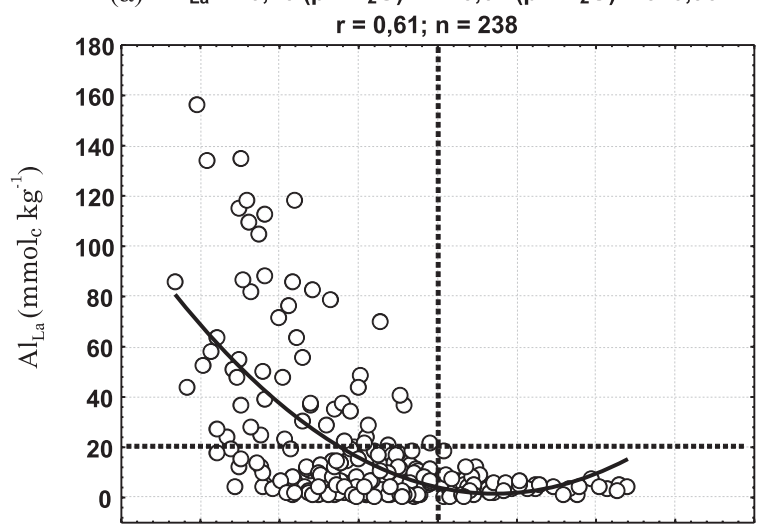

(b) $\mathrm{Al}_{\mathrm{Cu}}=-0,55\left(\mathrm{pH} \mathrm{H}_{2} \mathrm{O}\right)^{2}-22,06\left(\mathrm{pH} \mathrm{H}_{2} \mathrm{O}\right)+171,99$ $r=0,24 ; n=237$

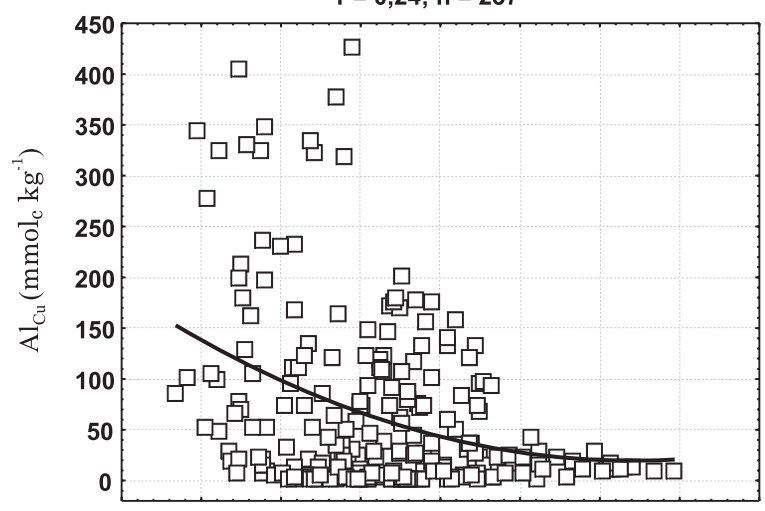

(c) $\mathrm{Al}_{\mathrm{K}}=4,00\left(\mathrm{pH} \mathrm{H}_{2} \mathrm{O}\right)^{2}-43,92\left(\mathrm{pH} \mathrm{H}_{2} \mathrm{O}\right)+121,86$ $r=0,69 ; n=232$

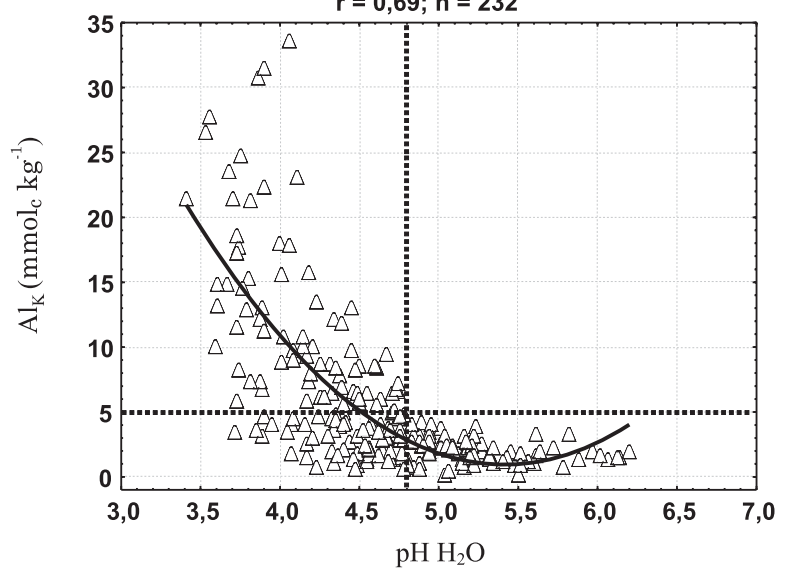

Figura 4. Relação entre os valores de pH medido em $\mathrm{H}_{2} \mathrm{O}\left(\mathrm{pH} \mathrm{H} \mathrm{H}_{2} \mathrm{O}\right)$ e as seguintes variáveis: $\mathrm{Al}_{\mathrm{La}}$ (a), $\mathrm{Al}_{\mathrm{Cu}}$ (b) e $\mathrm{Al}_{\mathrm{K}}$ (c). Linhas tracejadas indicam que em valor de $\mathrm{pH}$ superior a 5,0 os valores de $\mathrm{Al}_{\mathrm{La}}$ não ultrapassam $20 \mathrm{mmol}_{\mathrm{c}} \mathrm{kg}^{-1}$ e que acima de 4,8 a quantidade de $\mathrm{Al}_{\mathrm{K}}$ extraído é muito baixa, inferior a $5 \mathrm{mmol}_{\mathrm{c}} \mathrm{kg}^{-1}$. Valores relativos a todos os horizontes estudados com conteúdos de $\mathrm{Al}$ diferentes de zero nos diferentes extratores utilizados. 
Quadro 3. Coeficiente de correlação r do momento do produto Pearson e número de amostras n na relação linear entre a CTC do solo e as seguintes variáveis: $\mathrm{Al}_{\mathrm{p}}, \mathrm{Al}_{\mathrm{Cu}}, \mathrm{Al}_{\mathrm{La}}$ e $\mathrm{Al}_{\mathrm{K}}$. Valor de $\mathrm{p}<0,0001$ em todas as correlações

\begin{tabular}{|c|c|c|c|c|c|c|c|c|}
\hline \multirow{2}{*}{ Variável } & \multicolumn{2}{|c|}{$\mathrm{Al}_{\mathrm{p}}$} & \multicolumn{2}{|c|}{$\mathrm{Al}_{\mathrm{Cu}}$} & \multicolumn{2}{|c|}{$\mathrm{Al}_{\mathrm{La}}$} & \multicolumn{2}{|c|}{$\mathrm{Al}_{\mathbf{K}}$} \\
\hline & $\mathbf{r}$ & $\bar{n}$ & $\mathbf{r}$ & $\mathbf{n}$ & $\mathbf{r}$ & $\mathbf{n}$ & $\mathbf{r}$ & $\overline{\mathbf{n}}$ \\
\hline \multirow{4}{*}{ CTC } & \multicolumn{8}{|c|}{ Todos os horizontes } \\
\hline & 0,43 & 169 & 0,76 & 233 & 0,83 & 234 & 0,77 & 229 \\
\hline & \multicolumn{8}{|c|}{ Horizontes B espódicos } \\
\hline & 0,40 & 103 & 0,77 & 105 & 0,86 & 100 & 0,69 & 103 \\
\hline
\end{tabular}

Al-húmus, possivelmente regulando a atividade do elemento dissolvido nos Espodossolos sob vegetação de restinga do litoral paulista. A correlação entre CTC e Corg, com valores de $\mathrm{r}=0,86^{* * *}$ em todas as amostras e $\mathrm{r}=0,98^{* * *}$ em apenas nos horizontes B espódicos, evidencia que essas cargas resultam predominantemente da MOS.

Os resultados deste trabalho discordam daqueles encontrados por García-Rodeja et al. (2004). Esses autores determinaram que $\mathrm{Al}_{\mathrm{Cu}}$ foi o que melhor se correlacionou com CTC medida a pH 7 em horizontes ândicos. Possivelmente, o maior conteúdo e tipo diferenciado de minerais não cristalinos de $\mathrm{Al}$ naqueles solos em relação aos aqui estudados, os quais são parcialmente solúveis em $\mathrm{CuCl}_{2}$, mas não em $\mathrm{LaCl}_{3}$, são os responsáveis pela diferença de resultados. Os dados mostrados no quadro 4 parecem corroborar essa hipótese. Quando relacionamos as diferentes formas de $\mathrm{Al}$ (incluindo aquelas que representam graus variados de estabilidade da interação C-metal) com Corg e CTC de amostras que reconhecidamente apresentam minerais amorfos na sua constituição $\left(\mathrm{Al}_{\mathrm{p}} / \mathrm{Corg}>20\right)$, as melhores correlações se dão para $\mathrm{Al}_{\mathrm{Cu}}$ e $\mathrm{Al}_{\mathrm{Cu}}-\mathrm{Al}_{\mathrm{La}}$ (Quadro 3). Possivelmente, isso ocorre devido aos seguintes fatores: (a) íntima relação entre o Corg e os minerais amorfos dessas amostras; (b) contribuição desses minerais na CTC do solo; e (c) sua solubilidade parcial, ou da interação Corg-minerais amorfos, ao $\mathrm{CuCl}_{2}$, o que pode ser avaliado pelas relações significativas entre $\mathrm{Al}_{0}-\mathrm{Al}_{\mathrm{p}}$ e as variáveis $\mathrm{Al}_{\mathrm{Cu}}$ $\mathrm{e} \mathrm{Al}_{\mathrm{Cu}}-\mathrm{Al} \mathrm{La}_{\mathrm{La}}$.
A diferença $\mathrm{Al}_{0}-\mathrm{Al}_{\mathrm{p}}$ refere-se ao $\mathrm{Al}$ na estrutura dos minerais amorfos (García-Rodeja et al., 2004). As seguintes correlações significativas são observadas entre essa variável e as demais mostradas no quadro 4 , segundo uma ordem crescente de dispersão dos dados: $\mathrm{Al}_{\mathrm{o}}\left(\mathrm{r}=0,81^{* * *}\right), \mathrm{Al}_{\mathrm{p}}-\mathrm{Al}_{\mathrm{Cu}}\left(\mathrm{r}=0,61^{* * *}\right), \mathrm{Al}_{\mathrm{p}}(\mathrm{r}=$ $\left.0,60^{* * *}\right), \mathrm{Al}_{\mathrm{Cu}}-\mathrm{Al}_{\mathrm{La}}\left(\mathrm{r}=0,47^{* * *}\right)$ e $\mathrm{Al}_{\mathrm{Cu}}\left(\mathrm{r}=0,45^{* * *}\right)$, sugerindo que os extratores oxalato, pirofosfato e, menos eficientemente, $\mathrm{CuCl}_{2}$ removeram algum $\mathrm{Al}$ da estrutura desses minerais. No entanto, $\mathrm{LaCl}_{3} \mathrm{e}$ $\mathrm{KCl}$ não se relacionaram $(\mathrm{p}>0,01)$ com a diferença $\mathrm{Al}_{\mathrm{o}}-\mathrm{Al}_{\mathrm{p}}$, evidenciando-se suas especificidades para formas orgânicas e reativas de $\mathrm{Al}$, já que $\mathrm{Al}_{\mathrm{La}}$ e $\mathrm{Al}_{\mathrm{K}}$ mostram-se com elevada correlação com a CTC e Corg das amostras do quadro 4. No entanto, a boa correlação entre as formas mais estáveis da associação Corg-metal $\left(\mathrm{Al}_{\mathrm{p}}-\mathrm{Al}_{\mathrm{Cu}}\right)$ e o conteúdo de $\mathrm{Al}_{\mathrm{Cu}}(\mathrm{r}=0,70$; $\mathrm{p}<0,0001 ; \mathrm{n}=160)$ em todas as amostras estudadas indica que o $\mathrm{CuCl}_{2}$ extrai parte de compostos bastante estáveis da associação, possivelmente não reativos em termos de acidez do solo e troca iônica. Os extratores $\mathrm{LaCl}_{3}$ e $\mathrm{KCl}$ não mostraram correlações significativas com essas formas estáveis nas mesmas amostras $(\mathrm{r}=$ $0,05^{\mathrm{ns}} ; \mathrm{n}=160$ em ambos), corroborando suas especificidades a compostos organoalumínicos reativos.

As porcentagens do $\mathrm{Al}$ extraído com $\mathrm{LaCl}_{3}$ em relação ao pirofosfato e $\mathrm{CuCl}_{2}$ variaram de 1,1 até 125,5 \% (média de 29,4 \%) e de 3,4 até 151,5 \% (média de $46,2 \%)$, respectivamente, valores máximos e médios muito superiores àqueles relatados por Urrutia et al. (1995) e García-Rodeja et al. (2004), evidenciandose a presença de frações orgânicas de $\mathrm{Al}$ mais lábeis nos solos aqui estudados.

A máxima eficácia do $\mathrm{LaCl}_{3}$ em relação ao pirofosfato e ao $\mathrm{CuCl}_{2}$ para extrair $\mathrm{Al}$ associado à matéria orgânica ocorre, sobretudo, nos horizontes superficiais do tipo A, havendo tendência de sua redução em profundidade naqueles horizontes de maior estabilidade da interação Al-húmus. Em termos gerais, a relação entre a eficácia do $\mathrm{LaCl}_{3}$ na extração do $\mathrm{Al}$ e o tipo de horizonte dos solos estudados segue a ordem: $\mathrm{A}>\mathrm{Bh} \geq \mathrm{C}>\mathrm{Bhm}>\mathrm{Bs} / \mathrm{Bhs} / \mathrm{Bsm}$ (Figura 5). Assim, em um mesmo perfil, as menores eficácias foram observadas, em geral, em horizontes dotados de subscrito "s", em alguns horizontes Bhm de

Quadro 4. Coeficientes de correlação r, número de amostras n e níveis de significância p do momento do produto Pearson para a relação entre Corg, $\mathrm{CTC}$ e $\mathrm{Al}_{\mathrm{o}}-\mathrm{Al}_{\mathrm{p}}$ e as seguintes variáveis: $\mathrm{Al}_{\mathrm{o}}, \mathrm{Al}_{\mathrm{p}}, \mathrm{Al}_{\mathrm{Cu}}, \mathrm{Al}_{\mathrm{La}}, \mathrm{Al}_{\mathrm{K}}$, $\mathrm{Al}_{\mathrm{p}}-\mathrm{Al}_{\mathrm{Cu}}, \mathrm{Al}_{\mathrm{Cu}}-\mathrm{Al}_{\mathrm{La}}$ e $\mathrm{Al}_{\mathrm{La}}-\mathrm{Al}_{\mathrm{K}}$. Conjunto de dados com valores de ( $\left.\mathrm{Al}_{\mathrm{p}} / \mathrm{Corg}\right) 100$ iguais ou superiores a 20 e diferenças $\mathrm{Al}_{\mathrm{o}}-\mathrm{Al}_{\mathrm{p}}$ positivas

\begin{tabular}{|c|c|c|c|c|c|c|c|c|c|c|c|c|c|c|c|c|c|}
\hline \multirow{2}{*}{ Variável } & \multirow{2}{*}{$\mathbf{N}$} & \multicolumn{2}{|r|}{$\mathrm{Al}_{\mathrm{o}}$} & \multicolumn{2}{|r|}{$\mathrm{Al}_{\mathbf{p}}$} & \multicolumn{2}{|r|}{$\mathrm{Al}_{\mathrm{Cu}}$} & \multicolumn{2}{|r|}{$\mathrm{Al}_{\mathrm{La}}$} & \multicolumn{2}{|r|}{$\mathrm{Al}_{\mathrm{K}}$} & \multicolumn{2}{|c|}{$\mathrm{Al}_{\mathrm{p}-} \mathrm{Al}_{\mathrm{Cu}}$} & \multicolumn{2}{|c|}{$\mathrm{Al}_{\mathrm{Cu}-} \mathrm{Al}_{\mathrm{La}}$} & \multicolumn{2}{|c|}{$\mathrm{Al}_{\mathrm{La}-} \mathbf{A l}_{\mathrm{K}}$} \\
\hline & & $\mathbf{r}$ & $\mathbf{p}$ & $\mathbf{r}$ & $\mathbf{p}$ & $\mathbf{r}$ & $\mathbf{p}$ & $\mathbf{r}$ & $\mathbf{p}$ & $\mathbf{r}$ & $\mathbf{p}$ & $\mathbf{r}$ & $\mathbf{p}$ & $\mathbf{r}$ & $\mathbf{p}$ & $\mathbf{r}$ & $\mathbf{p}$ \\
\hline Corg & 46 & 0,74 & $<0,0001$ & 0,84 & $<0,0001$ & 0,93 & $<0,0001$ & 0,82 & $<0,0001$ & 0,79 & $<0,0001$ & 0,72 & $<0,0001$ & 0,92 & $<0,0001$ & 0,76 & $<0,0001$ \\
\hline CTC & 45 & 0,77 & $<0,0001$ & 0,79 & $<0,0001$ & 0,91 & $<0,0001$ & 0,82 & $<0,0001$ & 0,82 & $<0,0001$ & 0,66 & $<0,0001$ & 0,90 & $<0,0001$ & 0,75 & $<0,0001$ \\
\hline $\mathrm{Al}_{\mathrm{o}}-\mathrm{Al}_{\mathrm{p}}$ & 41 & 0,81 & $<0,0001$ & 0,60 & $<0,0001$ & 0,45 & 0,0041 & 0,24 & 0,1468 & 0,20 & 0,2284 & 0,61 & $<0,0001$ & 0,47 & 0,0028 & 0,24 & 0,1371 \\
\hline
\end{tabular}




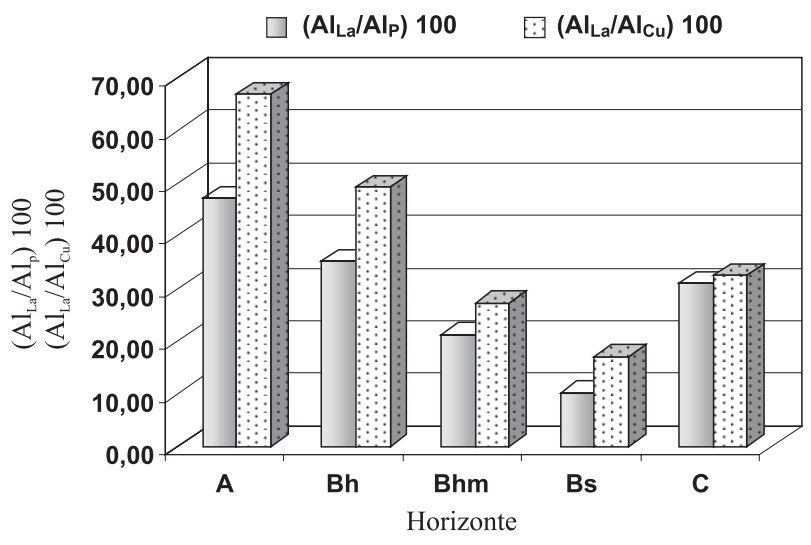

Figura 5. Conteúdos médios das relações $\left(\mathrm{Al}_{\mathrm{La}} / \mathrm{Al}_{\mathrm{p}}\right)$ 100 e $\left(\mathrm{Al}_{\mathrm{La}} / \mathrm{Al}_{\mathrm{Cu}}\right) 100$ por horizontes estudados, as quais refletem a eficácia do $\mathrm{LaCl}_{3}$ em relação aos demais extratores. Horizontes $A(n=16)$ incluem aqueles intermediários $\mathrm{AB}, \mathrm{AE}$ e $\mathrm{AC}$. Horizontes Bs $(n=48)$ referem-se aos descritos como Bs, Bhs e Bsm. Horizontes $\mathrm{C}(\mathrm{n}=36)$ incluem os intermediários CA e CB. Horizontes Bh e Bhm com número de amostras (n) de 41 e 13, respectivamente. Média dos valores diferentes de zero.

determinados perfis, bem como em alguns horizontes C bem drenados e com baixos conteúdos de Corg (inferior a $10 \mathrm{~g} \mathrm{~kg}^{-1}$ ), os quais mostraram a maior estabilidade da interação Al-húmus (Quadro 1).

Esses resultados corroboram os discutidos anteriormente e, em parte, àqueles relatados por Urrutia et al. (1995). Segundo esses autores, as menores eficácias do $\mathrm{LaCl}_{3}$ ocorreram em Andossolos e, à semelhança do que se encontrou aqui, em alguns horizontes Bs e C de Espodossolos com baixos conteúdos de Corg, em que o extrator somente extraiu Al trocável (Urrutia et al., 1995). Alguns horizontes Bs e C bem drenados aqui estudados comprovadamente apresentam formas inorgânicas de $\mathrm{Al}$ não cristalinas, as quais são solúveis em pirofosfato e $\mathrm{CuCl}_{2}$, mas não em $\mathrm{LaCl}_{3}$, diminuindo sua eficácia relativa a esses extratores (Coelho et al., 2010b).

Dados de perfis selecionados do quadro 1 corroboram as assertivas anteriores. Observa-se, nesse quadro, que a maioria dos horizontes $\mathrm{C}$ de perfis bem drenados (Perfis P1 e P5) apresentou valores de $\mathrm{pH}$ próximos a 5 e relação $\mathrm{Al}_{\mathrm{p}} / \mathrm{Al}_{0}$ inferior à unidade, indicativos de condições favoráveis à formação $(\mathrm{pH}) \mathrm{e}$ presença de minerais de $\mathrm{Al}$ não cristalinos $\left(\mathrm{Al}_{\mathrm{p}} / \mathrm{Al}_{\mathrm{o}}\right)$. Nesses horizontes com baixo conteúdo de Corg, a eficácia do $\mathrm{LaCl}_{3}$ em relação ao pirofosfato foi baixa (< $7 \%$ ), possivelmente devido a uma conjugação de dois fatores: (1) habilidade deste último para formas inorgânicas de Al; e, sobretudo, (2) maior estabilidade da interação Al-húmus de tais horizontes.

Em horizontes $\mathrm{C}$ mal drenados, como aqueles do perfil P8 (Quadro 1), a eficácia do $\mathrm{LaCl}_{3}$ para formas de $\mathrm{Al}$ é semelhante ou, mesmo, superior à do pirofosfato e $\mathrm{CuCl}_{2}$, indicativo da presença de formas lábeis da interação Al-húmus, possivelmente condicionadas pelo hidromorfismo (García-Rodeja et al., 2004). Nesses horizontes, os baixos valores de $\mathrm{pH}(<3,4)$ favorecem a interação Al-húmus em detrimento da formação de compostos inorgânicos de Al (Shoji \& Fujiwara, 1984). Fato semelhante ocorreu em alguns poucos horizontes A (Perfil P10). Embora esses horizontes fossem bem drenados, a constante deposição de matéria orgânica fresca pouco humificada, os baixos conteúdos de $\mathrm{Al}$ total (Hargrove \& Thomas, 1984) e valores de $\mathrm{pH}$ (Jarvis, 1986) favoreceram a formação de espécies de Al menos hidroxiladas e polimerizadas, nas quais o $\mathrm{LaCl}_{3}$ foi muito efetivo na sua remoção (Hargrove \& Thomas, 1984), sobrepondo-se à capacidade extratora do pirofosfato e $\mathrm{CuCl}_{2}$.

Os horizontes Bs e Bhs do perfil P8 permanecem inundados a maior parte do tempo ao longo dos anos e não mostram indícios de materiais inorgânicos não cristalinos, como pode ser concluído dos baixos valores de $\mathrm{pH}(<3,6)$ e das relações $\mathrm{Al}_{\mathrm{p}} / \mathrm{Al}_{\mathrm{o}}$ próximas à unidade. Seus valores de $\mathrm{Al}$ extraído por $\mathrm{LaCl}_{3}$ em relação aos extratores pirofosfato e $\mathrm{CuCl}_{2}$ foram muito superiores àqueles dos perfis bem drenados (P5, P29 e P31), o que também sugere a manutenção de formas mais lábeis de $\mathrm{Al}$ sob condições de hidromorfismo.

Dos enunciados acima se pode argumentar que a extração com $\mathrm{LaCl}_{3}$ pode ser interpretada em termos da estabilidade dos complexos Al-húmus (GarcíaRodeja et al., 2004), de tal forma que nos horizontes Bs, Bhs, Bsm, Bhm e C (bem drenados) ocorrem as menores eficácias do extrator para formas orgânicas de $\mathrm{Al}$, enquanto em alguns desses horizontes, sobretudo Bs, Bhs e C, se encontrados sob condições frequentes de hidromorfismo, o extrator tem sua eficácia aumentada devido à presença e manutenção de matéria orgânica menos condensada e formas de $\mathrm{Al}$ menos polimerizadas. Horizontes superficiais do tipo A geralmente possuem matéria orgânica mais lábil de um perfil e, portanto, maior eficácia relativa do $\mathrm{LaCl}_{3}$ em relação ao pirofosfato e $\mathrm{CuCl}_{2}$ (Quadro 1).

\section{Al extraído com $\mathrm{KCl}\left(\mathrm{Al}_{\mathrm{K}}\right)$ e sua comparação ao $\mathrm{Al}_{\mathrm{Cu}}$ e $\mathrm{Al}_{\mathrm{La}}$}

A percentagem de $\mathrm{Al}$ extraído por $\mathrm{KCl}$ em comparação com o $\mathrm{Al}_{\mathrm{p}}$ foi de 0,3 a $63,0 \%$, com média de $10,0 \%$. No entanto, a fração de $\mathrm{Al}_{\mathrm{La}}$ extraída por $\mathrm{KCl}$ foi bem mais elevada, com os valores mínimo e máximo e a média de 7,$5 ; 300,0$; e 53,1\%, respectivamente. Em alguns poucos horizontes superficiais (A, AE, EA e E) e horizontes $\mathrm{C}$, o $\mathrm{KCl}$ foi mais efetivo na remoção de $\mathrm{Al}$ que o $\mathrm{LaCl}_{3}$, evidenciando-se formas mais lábeis de $\mathrm{Al}$ nesses horizontes em relação aos B espódicos. Esses últimos tiveram os maiores conteúdos de $\mathrm{Al}_{\mathrm{K}}$ removidos, com valores mínimos e máximos de 1,69 e 33,50 mmol $_{\mathrm{c}} \mathrm{kg}^{-1}$, respectivamente, e média de $8,87 \mathrm{mmol}_{\mathrm{c}} \mathrm{kg}^{-1}$, enquanto nos horizontes superficiais do tipo $\mathrm{A}$ os 
mesmos valores foram inferiores, respectivamente 0,5 ; 23,5; e 5,98 $\mathrm{mmol}_{\mathrm{c}} \mathrm{kg}^{-1}$. Considerando todos os horizontes estudados, os valores mínimos e máximos do $\mathrm{Al}_{\mathrm{K}}$ foram de 0,10 ; e 33,50 , com média de $5,81 \mathrm{mmol}_{\mathrm{c}} \mathrm{kg}^{-1}$.

Similarmente ao observado com o $\mathrm{CuCl}_{2}$ (Figura 4a) e o $\mathrm{LaCl}_{3}$ (Figura 4b), existe relação inversa entre o $\mathrm{pH}$ medido em água e o $\mathrm{Al}$ extraído por $\mathrm{KCl}$ $1 \mathrm{~mol} \mathrm{~L}^{-1}$ (Figura 4c). O melhor ajuste das curvas, no entanto, ocorre com esse extrator, quer relacionando o $\mathrm{Al}_{\mathrm{K}}$ ao $\mathrm{pH}$ medido em água $(\mathrm{r}=-0,69$; curva polinomial; figura 4), quer relacionando-o à solução de equilíbrio ( $r=-0,82$; curva logarítmica; figura 6a). Esses resultados podem ser discutidos à luz da capacidade-tampão dos reagentes utilizados e quanto à afinidade dos cátions aos sítios de troca da MOS; o $\mathrm{CuCl}_{2}$ em comparação ao $\mathrm{KCl}$ e $\mathrm{LaCl}_{3}$ é tamponado. A solução de $\mathrm{CuCl}_{2}$ é um ácido fraco ( $\mathrm{pH} 3$ ) e, portanto, eficiente agente tamponante, controlando e definindo o $\mathrm{pH}$ final da mistura solo-solução (Oates \& Kamprath, 1983a).

Ainda que a relação entre o pH de equilíbrio e o conteúdo de $\mathrm{Al}$ extraído com $\mathrm{CuCl}_{2}$ da figura 6 a seja significativa, a dispersão dos dados é muito alta $(\mathrm{r}=$ $0,63 * * *)$, indicando que apenas o pH não explica a eficácia desse extrator para formas de $\mathrm{Al}$ nos solos estudados. $\mathrm{O}$ poder de substituição do $\mathrm{CuCl}_{2}$ também pode ser atribuído à forte afinidade dos íons $\mathrm{Cu}$ pelos sítios de troca orgânicos, tal como afirmaram Hargrove \& Thomas (1984). Entretanto, o $\mathrm{LaCl}_{3}$ tem seu efeito atribuído, sobretudo, à afinidade do cátion aos sítios de troca do solo (Bloom et al., 1979b), como pode ser observado pela grande dispersão dos dados da figura $6 \mathrm{~b}$, mesmo com baixos valores de $\mathrm{pH}$ de equilíbrio (média de 2,7), mais próximos daqueles observados em $\mathrm{CuCl}_{2}$ (valores mínimo e máximo e a média de 1,$96 ; 3,66$; e 2,68 , respectivamente), comparativamente ao $\mathrm{KCl}(\mathrm{pH}$ mínimo de 2,88, máximo de 6,13 e média de 4,59). Com esse último extrator, parece que o $\mathrm{pH}$ da mistura de solução salina e solo no final da extração teve efeito expressivo no conteúdo de $\mathrm{Al}$ extraído ( $\mathrm{r}=-0,82$, curva exponencial; Figura 6c), fato também constatado por Ponette et al. (1996). No entanto, algum efeito do cátion é esperado, sobretudo a valores de $\mathrm{pH}$ no extrato de equilíbrio inferiores a 4,5 .

Pelo exame da figura $6 c$, nota-se que a maior dispersão dos dados e os maiores conteúdos de $\mathrm{Al}$ trocável ocorrem a valores de $\mathrm{pH}$ de equilíbrio inferiores a 4,5, sugerindo que espécies de $\mathrm{Al}^{3+}$ podem estar presentes no complexo de troca, as quais são mais facilmente trocadas pelo potássio que espécies hidróxi $\left(\mathrm{Al}(\mathrm{OH})^{2+}\right.$ e $\left.\mathrm{Al}(\mathrm{OH})_{2}{ }^{+}\right)$; estas predominam em $\mathrm{pH}$ superior a 4,5 e tendem a se polimerizar, tornando difícil de serem substituídas por um simples cátion de troca (Jarvis, 1986), sobretudo se monovalente, como o potássio (Ponette et al., 1996). De fato, valores de $\mathrm{pH}$ próximos ou superiores a 5,0 correspondem ou (1) a horizontes com muito baixos a não detectáveis
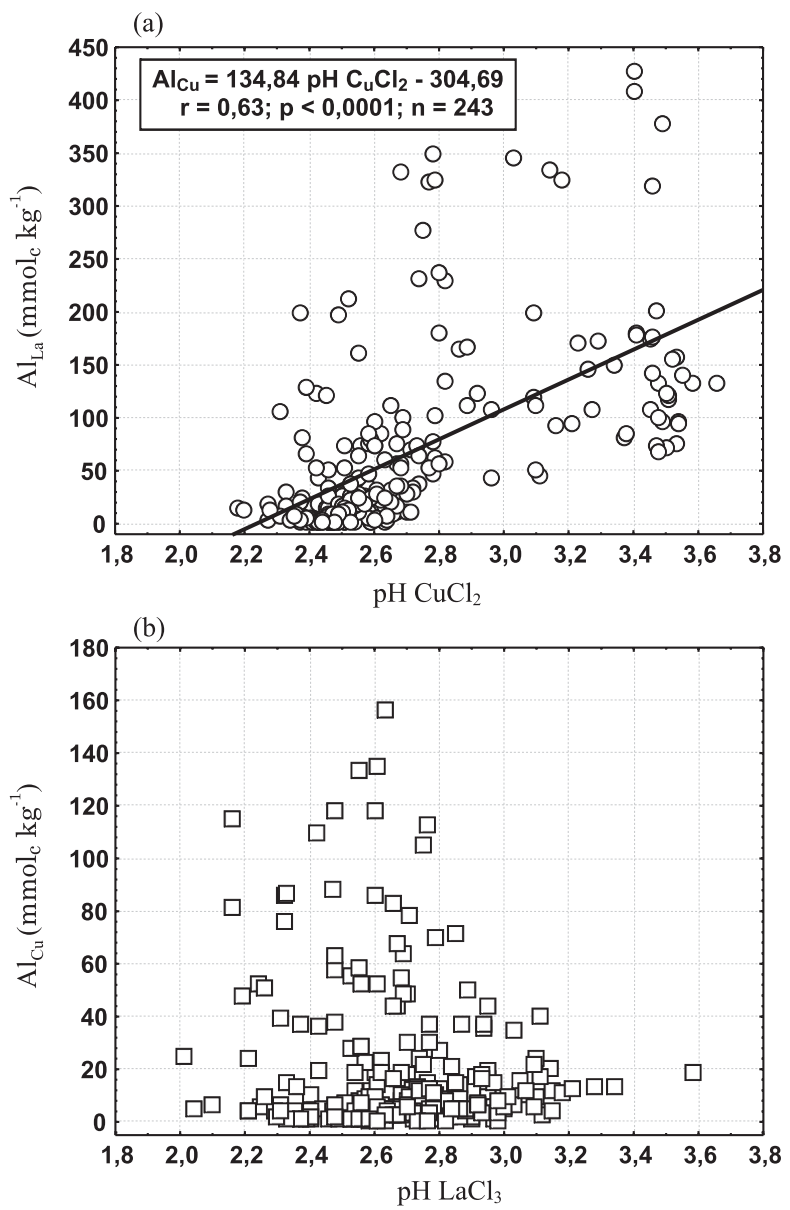

(c)

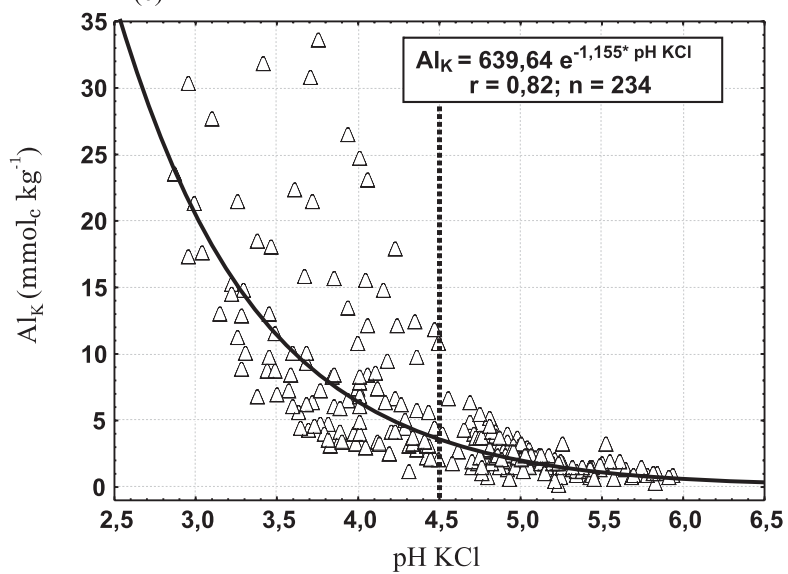

Figura 6. Relação entre os teores de $\mathrm{Al}$ extraídos com (a) $\mathrm{CuCl}_{2}\left(\mathrm{Al}_{\mathrm{Cu}}\right)$, (b) $\mathrm{LaCl}_{3}\left(\mathrm{Al}_{\mathrm{La}}\right)$ e (c) $\mathrm{KCl}\left(\mathrm{Al}_{\mathrm{K}}\right)$ e os respectivos valores do $\mathrm{pH}$ medidos em solução de equilíbrio ( $\mathrm{pH} \mathrm{CuCl} \mathrm{Cu}_{2} \mathrm{paCl}_{3}$ e pH KCl). Linha tracejada indica o valor de $\mathrm{pH}$ de 4,5 na solução de equilíbrio do extrator $\mathrm{KCl}(\mathrm{pH} \mathrm{KCl})$, abaixo do qual há maior dispersão dos dados.

conteúdos de Corg $\left(<6,00 \mathrm{~g} \mathrm{~kg}^{-1}\right)$, como horizontes $\mathrm{E}$ e intermediários (EA e EB), ou (2) a alguns horizontes $\mathrm{C}$, Bhs e Bs bem drenados, predominantemente também com baixos conteúdos de Corg $\left(<14 \mathrm{~g} \mathrm{~kg}^{-1}\right)$, como se pode observar no quadro 1. Enquanto os 
primeiros mostraram os menores valores de $\mathrm{Al}$ obtido de todos os extratores em solos aqui estudados, os B espódicos e $\mathrm{C}$ bem drenados supracitados têm seu baixo conteúdo de $\mathrm{Al}$ extraído por $\mathrm{KCl}$ devido à predominância de complexos Al-húmus menos lábeis.

A figura 7 evidencia a relação entre o Corg e o $\mathrm{pH}$ de equilíbrio obtido dos diferentes extratores e amostras. O melhor ajuste das curvas se dá quando se analisam as soluções extraídas com $\mathrm{KCl}(\mathrm{r}=0,78$; curva exponencial; Figura 7c), sendo muito pior ajustada nos demais extratores $\left(r=0,39\right.$ em $\mathrm{LaCl}_{3}$, curva exponencial e $\mathrm{r}=0,25$ em $\mathrm{CuCl}_{2}$, curva polinomial). Do exame da referida figura, pode-se sugerir que: (a) A maior amplitude dos valores de $\mathrm{pH} \mathrm{KCl} \mathrm{em} \mathrm{relação} \mathrm{aos} \mathrm{de} \mathrm{pH}$ de equilíbrio dos demais extratores se deve ao seu menor poder tamponante. Assim, o pH da mistura solo-solução extratora, quando se utiliza o $\mathrm{KCl}$, será fortemente influenciado pelo $\mathrm{pH}$ do solo. De fato, a relação entre o $\mathrm{pH}$ do solo medido em água e o $\mathrm{pH}$ de equilíbrio é significativa e a curva é mais bem ajustada quando se compara $\mathrm{KCl}\left(\mathrm{pH} \mathrm{H}_{2} \mathrm{O}\right.$ $\left.\mathrm{x} \mathrm{pH} \mathrm{KCl} ; \mathrm{r}=0,74^{* * *}\right)$ ao $\mathrm{LaCl}_{3}\left(\mathrm{pH} \mathrm{H} \mathrm{H}_{2} \mathrm{O} \times \mathrm{pH} \mathrm{LaCl}{ }_{3}\right.$; $\left.\mathrm{r}=0,37^{* * *}\right)$ e $\mathrm{CuCl}_{2}\left(\mathrm{pH} \mathrm{H} \mathrm{H}_{2} \mathrm{O} \times \mathrm{pH} \mathrm{CuCl} 2 ; \mathrm{r}=0,06\right)$, sendo este último, portanto, o reagente de maior capacidade-tampão; (b) a matéria orgânica foi fonte de acidez trocável nos solos estudados quando se utilizaram extratores com baixa capacidade de tamponamento. Por conseguinte, (c) os extratores considerados não tamponados, como $\mathrm{CuCl}_{2}$ e $\mathrm{LaCl}_{3}$, cuja efetividade se deve à grande afinidade do cátion aos sítios de troca da MOS, extraem acidez outra que não apenas as trocáveis por $\mathrm{KCl} 1 \mathrm{~mol} \mathrm{~L}^{-1}$, e suas capacidades de tamponamento são diferenciadas e consideravelmente superiores em relação à desse extrator.

Somente horizontes com $\mathrm{pH}\left(\mathrm{H}_{2} \mathrm{O}\right)$ inferiores a 4,8 e 4,5 tiveram, respectivamente, $\mathrm{Al}_{\mathrm{K}}$ maior que 5 e $10 \mathrm{mmol}_{\mathrm{c}} \mathrm{kg}^{-1}$ (Figura 4c). Conteúdos de $\mathrm{Al}_{\mathrm{K}}$ maiores que $30 \mathrm{mmol}_{\mathrm{c}} \mathrm{kg}^{-1}$ foram obtidos de poucos horizontes Bh muito ácidos ( $\mathrm{pH} \mathrm{H}_{2} \mathrm{O}<4,1$; Figura 4c), o que está de acordo com o caráter lábil do elemento nesses horizontes e seu maior conteúdo médio em relação àqueles superficiais, em que o $\mathrm{Al}$ também é lábil.

A figura 8a,c mostra a relação entre o $\mathrm{pH}$ medido em água $\left(\mathrm{pH} \mathrm{H}_{2} \mathrm{O}\right)$ e os conteúdos de $\mathrm{Al}$ unidos à matéria orgânica em diferentes graus de estabilidade. Nota-se que há um deslocamento para faixas de $\mathrm{pH}$ cada vez mais baixas à medida que o $\mathrm{Al}$ se torna mais lábil $\left(\mathrm{Al}_{\mathrm{La}}-\mathrm{Al}_{\mathrm{K}}\right)$, evidenciando que tais espécies, provavelmente trocáveis, estão presentes e são as principais formas do elemento extraídas especificamente por $\mathrm{LaCl}_{3}\left(\mathrm{Al}_{\mathrm{La}}-\mathrm{Al}_{\mathrm{K}}\right)$ na faixa de $\mathrm{pH}$ compreendida entre 3,4 e 4,4. Em contrapartida, as de maior estabilidade da interação Al-húmus $\left(\mathrm{Al}_{\mathrm{p}}-\mathrm{Al}_{\mathrm{Cu}}\right)$ se concentram em valores de $\mathrm{pH}$ mais elevados, situados na faixa entre 4,1 e 5,5 (Figura 8a), cujos conteúdos extraídos foram os maiores aqui encontrados. No entanto, acima de pH 5,5 os valores de $\mathrm{Al}$ são baixos, inferiores a $50 \mathrm{mmol}_{\mathrm{c}} \mathrm{kg}^{-1}$, mesmo nas formas mais estáveis do
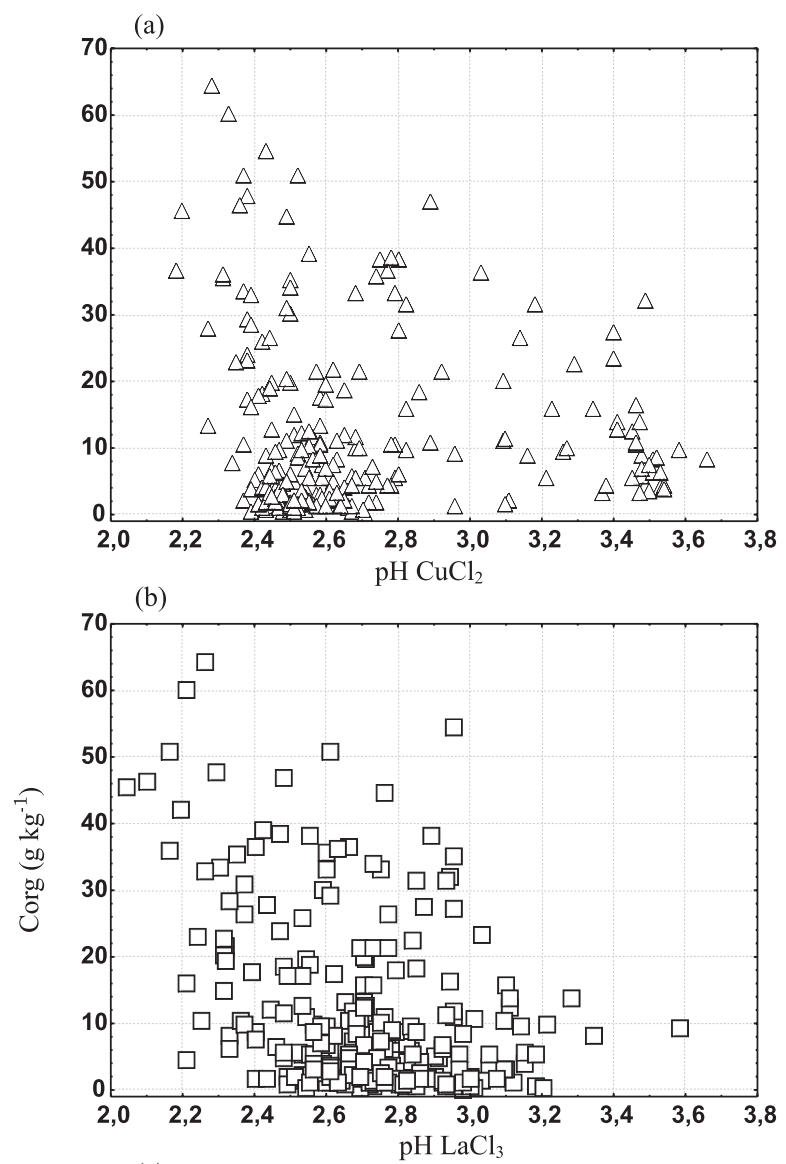

(c)

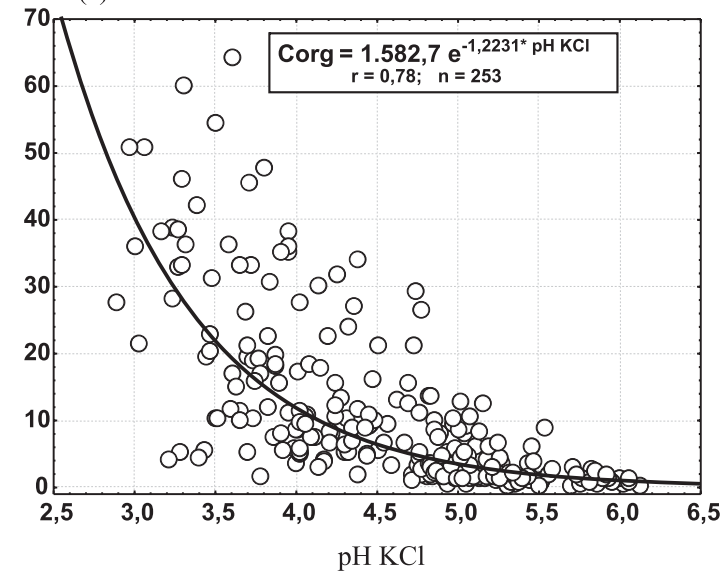

Figura 7. Relação entre o Corg e o pH de equilíbrio obtido com os extratores (a) $\mathrm{CuCl}_{2}(\mathrm{pH} \mathrm{CuCl})_{2}$, (b) $\mathrm{LaCl}_{3}(\mathrm{pH} \mathrm{LaCl})_{3}$ e (c) $\mathrm{KCl}(\mathrm{pH} \mathrm{KCl})$, em todas as amostras estudadas.

elemento $\left(\mathrm{Al}_{\mathrm{p}}-\mathrm{Al} \mathrm{Cu}_{\mathrm{Cu}}\right)$, correspondendo, sobretudo, a horizontes subsuperficiais do tipo $\mathrm{C}$ de perfis bem drenados, com baixos conteúdos de $\operatorname{Corg}\left(<5 \mathrm{~g} \mathrm{~kg}^{-1}\right) \mathrm{e}$ relação molar $\mathrm{Al}_{\mathrm{p}} / \mathrm{Al}_{\mathrm{o}}$ inferior a 1 , indicativo da presença de amorfos inorgânicos de $\mathrm{Al}$.

A influência do pH na estabilidade da interação Al-húmus dos solos estudados (Figura 8) também foi constatada por García-Rodeja et al. (2004) em solos vulcânicos europeus, de tal forma que horizontes 
extremamente ácidos, com $\mathrm{pH}<4,0$, detêm as formas mais lábeis de $\mathrm{Al}$, possivelmente com o elemento unido à superfície da matéria orgânica por meio de forças eletrostáticas. Acima desse valor, espécies de $\mathrm{Al}$ em

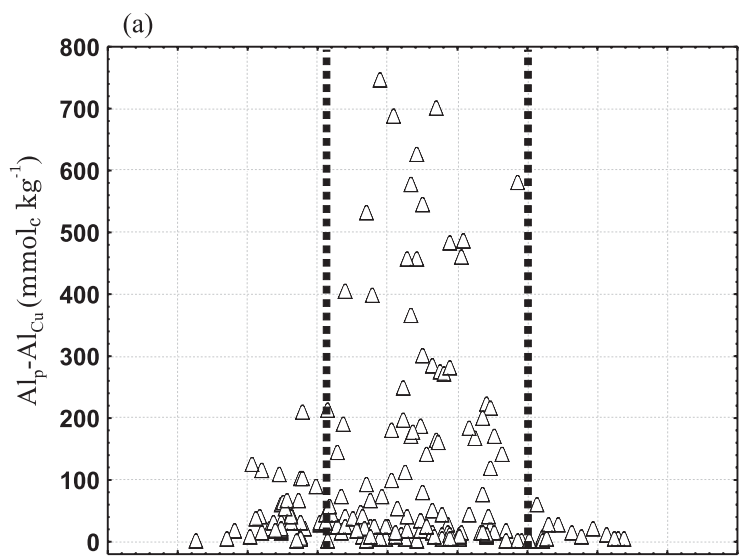

(b)

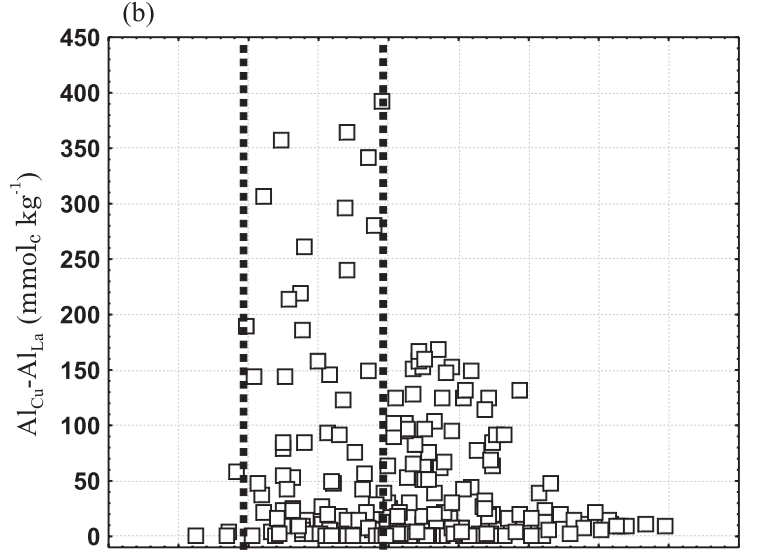

(c)

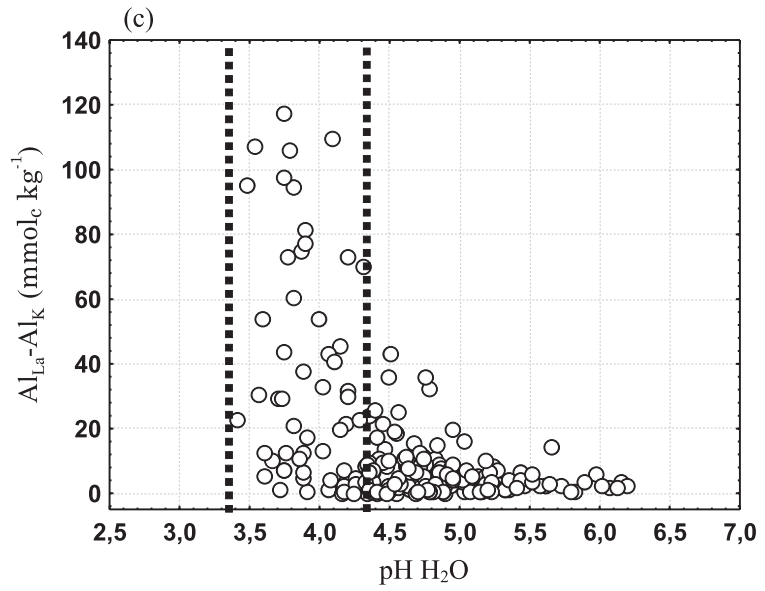

Figura 8. Relação entre o pH medido em água $\left(\mathrm{pH} \mathrm{H}_{2} \mathrm{O}\right)$ e as seguintes variáveis em ordem decrescente da estabilidade da interação $\mathrm{Al}$ húmus: (a) $\mathrm{Al}_{\mathrm{p}}-\mathrm{Al}_{\mathrm{Cu}}$; (b) $\mathrm{Al}_{\mathrm{Cu}}-\mathrm{Al}_{\mathrm{La}}$, (c) $\mathrm{Al}_{\mathrm{La}}-\mathrm{Al}_{\mathrm{K}}$. Linhas tracejadas correspondem às faixas de $\mathrm{pH}$ que englobam, entre outros valores, todos aqueles que representam no mínimo $50 \%$ do máximo valor de $\mathrm{Al}$ de cada diferença entre extratores. Utilizaram-se somente valores com diferenças positivas entre extratores. complexos orgânicos tornam-se cada vez mais abundantes e predominam em amostras que apresentam valores de $\mathrm{pH}$ entre 4,5 e 5,0, de tal forma que em condições mais alcalinas que $\mathrm{pH} 5,0$ o conteúdo das formas mais lábeis $\left(\mathrm{Al}_{\mathrm{La}}-\mathrm{Al}_{\mathrm{K}}\right.$; Figura $\left.8 \mathrm{c}\right)$ e trocáveis do elemento $\left(\mathrm{Al}_{\mathrm{K}}\right.$; Figura $\left.4 \mathrm{c}\right)$ é muito baixo, aquém de 20 e 5 mmol $_{\mathrm{c}} \mathrm{kg}^{-1}$, respectivamente.

\section{CONCLUSÕES}

1. O teor de $\mathrm{Al}$ extraído segue aquela comumente descrita na literatura: $\mathrm{Al}_{\mathrm{p}}>\mathrm{Al}_{\mathrm{Cu}}>\mathrm{Al}_{\mathrm{La}}>\mathrm{Al}_{\mathrm{K}}$.

2. Todas as formas de $\mathrm{Al}$ estudadas aumentaram com o conteúdo de $\mathrm{C}$ orgânico dos solos. No entanto, $\mathrm{o}$ $\mathrm{Al}_{\mathrm{La}}$ apresenta melhor correlação com essa variável, assim como com a CTC do solo, indicando que, dos reagentes analisados, foi o que melhor se relacionou aos componentes responsáveis pelo desenvolvimento de cargas nos solos estudados.

3. $\mathrm{O} \mathrm{CuCl}_{2}$, extrator reconhecidamente mais forte que o $\mathrm{LaCl}_{3}$ e menos eficaz que o pirofosfato, extrai parte do Al de formas amorfas inorgânicas e de complexos orgânicos bastante estáveis; esses últimos compreendem formas não ativas do elemento em termos de acidez do solo e troca iônica.

4. Ao contrário, a extração com $\mathrm{KCl}$ e $\mathrm{LaCl}_{3}$ inclui o $\mathrm{Al}$ reativo associado à matéria orgânica, e o $\mathrm{KCl}$ mostra sua máxima eficácia a valores de $\mathrm{pH}$ do solo inferiores a 4,5, devido à maior presença de espécies de $\mathrm{Al}^{3+}$.

5. Próximo e acima de $\mathrm{pH}$ 4,5 predominam espécies mais estáveis da interação Al-húmus, evidenciando que a acidez do solo controla sua formação e estabilidade, de tal forma que apenas a valores próximos ou superiores a 5,0 foram identificadas espécies de $\mathrm{Al}$ inorgânico não cristalino, sobretudo em horizontes Bs, Bhs e $\mathrm{C}$ bem drenados e com baixos conteúdos de Corg.

6. Tais horizontes (Bs, Bhs e C) apresentam as formas mais estáveis de $\mathrm{Al}$ complexado na matéria orgânica, seguidos do horizonte Bhm e, depois, do Bh. Os horizontes superficiais do tipo A, em geral, detêm as formas mais lábeis do elemento no perfil.

\section{LITERATURA CITADA}

BACHE, B.W. Aluminium mobilization in soils and waters. J. Geol. Soc., 143:699-706, 1986.

BLOOM, P.R.; MAcBRIDE, M.B. \& WEAVER, R.M. Aluminum organic matter in acid soils: Buffering and solution aluminum activity. Soil Sci. Soc. Am. J., 43:488-493, 1979a.

BLOOM, P.R.; MAcBRIDE, M.B. \& WEAVER, R.M. Aluminum organic matter in acid soils: Salt-extractable aluminum. Soil. Sci. Soc. Am. J., 43:488-493, 1979b. 
BUURMAN, P.; LAGEN, B. \& VELTHORST, E.J. Manual of soil and water analysis. Leiden, Backhuys Publishers Leiden, 1996. 314p.

COELHO, M.R.; MARTINS, V.M.; VIDAL-TORRADO, P.; OTERO-PÉREZ, X.L. \& VÁZQUEZ, F.M. Relação solorelevo-substrato geológico nas restingas da planície costeira do Estado de São Paulo. R. Bras. Ci. Solo, 34:833$846,2010 \mathrm{a}$

COELHO, M.R.; VIDAL-TORRADO, P.; OTERO-PÉREZ, X.L.; MARTINS, V.M. \& VÁZQUEZ, F.M. Fracionamento do $\mathrm{Al}$ por técnicas de dissoluções seletivas em Espodossolos da planície costeira do Estado de São Paulo. R. Bras. Ci. Solo, 34:1081-1092, 2010b.

COELHO, M.R.; VIDAL-TORRADO, P.; OTERO-PÉREZ, X.L.; MARTINS, V.M. \& VÁZQUEZ, F.M. Seletividade do pirofosfato de sódio e de cloretos não tamponados $\left(\mathrm{CuCl}_{2}\right.$ e $\mathrm{LaCl}_{3}$ ) como extratores de alumínio associado à matéria orgânica em solos de restinga do Estado de São Paulo. R. Bras. Ci. Solo, 34:1561-1572, 2010c.

DAHLGREN, R.A. \& WALKER, W.J. Aluminum release rates from selected Spodosol Bs horizons: Effect of $\mathrm{pH}$ and solid-phase aluminum pools. Geochem. Cosmoch. Acta, $57: 57-66,1993$

DAHLGREN, R.A. \& WALKER, W.J. Solubility control of KCl extractable aluminum in soils with variable charge. Comm. Soil Sci. Plant Anal., 25:2201-2214, 1994.

EMPRESA BRASILEIRA DE PESQUISA AGROCUPECUÁRIA - EMBRAPA. Centro Nacional de Pesquisa em Solos. Sistema brasileiro de classificação de solos. 2.ed. Rio de Janeiro, 2006. 306p.

EMPRESA BRASILEIRA DE PESQUISA AGROCUPECUÁRIA - EMBRAPA. Centro Nacional de Pesquisa em Solos. Manual de métodos de análise de solo. 2.ed. Rio de Janeiro, 1997. $212 \mathrm{p}$

GARCÍA-RODEJA, E.; NÓVOA, J.C.; PONTEVEDRA, X.; MARTÍNEZ-CORTIZAS, A. \& BUURMAN, P. Aluminium fraccionation of European volcanic soils by selective dissolution techniques. Catena, 56:155-183, 2004

GILLMAN, G.P. \& SUMPTER, E.A. KCl-extractable aluminium in highly weathered soils. Is it exchangeable? Comm. Soil Sci. Plant Anal., 16:561-568, 1985.

HARGROVE, W.L. \& THOMAS, G.W. Extraction of aluminum organic matter complexes. Soil Sci. Soc. Am. J., 45:151 153,1981

HARGROVE, W.L. \& THOMAS, G.W. Extraction of aluminum organic matter in relation to titratable acidity. Soil Sci. Soc. Am. J., 48:1458-1460, 1984.

HIGASHI, T.; DE CONINCK, F. \& GELAUDE, F. Characterization of some spodic horizons of the Campine (Belgium) with dithionite-citrate, pyrophosphate and sodium hydroxide-tetraborate. Geoderma, 25:131-142, 1981.

JARVIS, S.C. Forms of aluminium in some acid permanent grassland soils. J. Soil Sci., 37:211-222, 1986.
JEANROY, E. \& GUILLET, B. The occurrence of suspended ferruginous particles in pyrophosphate extracts of some soil horizons. Geoderma, 26:95-106, 1981.

JUO, A.S. \& KAMPRATH, E.J. Cooper chloride as an extractant for estimating the potentially reactive aluminium pool in acid soils. Soil Sci. Soc. Am. J., 43:35$38,1979$.

KAISER, K. \& ZECH, W. Defects in estimation of aluminum in humus complexes of Podzolic soils by pyrophosphate extraction. Soil Sci., 161:452-458, 1996.

LIN, C.L. \& COLEMAN, M.T. The measurement of exchangeable aluminum in soils. Soil Sci. Soc. Am. Proc., 24:444-446, 1960

MARTINS, S.E.; ROSSI, L.; SAMPAIO, P.S.P. \& MAGENTA, M.A.G. Caracterização florística de comunidades vegetais de restinga em Bertioga, SP, Brasil. Acta Bot. Bras., 22:249-274, 2008.

OATES, K.M. \& KAMPRATH, E.J. Soil acidity and liming: I. Effect of the extracting solution cation and $\mathrm{pH}$ on the removal of aluminum from acid soils. Soil Sci. Soc. Am. J., 47:686-689, 1983a.

OATES, K.M. \& KAMPRATH, E.J. Soil acidity and liming: II. Evaluation of using aluminum extracted by various chloride salts for determining lime requirements. Soil Sci. Soc. Am. J., 47:690-692, 1983b.

OLIVEIRA, J.B.; JACOMINE, P.K.T. \& CAMARGO, M.N. Classes gerais de solos do Brasil: Guia auxiliar para seu reconhecimento. 2.ed. Jaboticabal, FUNEP, 1992. 201p.

PATERSON, E.; CLARK, L. \& BIRNIE, C. Sequencial selective dissolution of iron, aluminium, and silicon from soils. Comm. Soil Sci. Plant Anal., 24:2015-2023, 1993.

PONETTE, Q.; ANDRE, D. \& DUFEY, J.E. Chemical significance of aluminium extracted from three horizons of an acid forest soil, using chloride alt solutions. Europ. J. Soil Sci., 47:89-95, 1996.

RAIJ, B.van; ANDRADE, J.C.; CANTARELLA, H. \& QUAGGIO, J.A. Análise química para avaliação da fertilidade de solos tropicais. Campinas, Instituto Agronômico de Campinas, 2001. 285p.

SANTOS, R.D.; LEMOS, R.C.; SANTOS, H.G.; KER, J.C. \& ANJOS, L.H.C. Manual de descrição e coleta de solo no campo. 5.ed. Viçosa, MG, Sociedade Brasileira de Ciência do Solo, 2005. 100p.

SAUER, D.; SPONAGEL, H.; SOMMER, M.; GIANI, L.; JAHN, R. \& STAHR, K. Review article - Podzol: Soil of the year 2007 - A review on its genesis, occurrence, and functions. J. Plant Nutr. Soil Sci., 170:581-597, 2007.

SETZER, J. Atlas climático e ecológico do Estado de São Paulo. São Paulo, Centrais Elétricas de São Paulo, 1966. 61p.

SHOJI, S. \& FUJIWARA, Y. Active aluminum and iron in the humus horizons of Andosols from northeastern Japan: Their forms, properties and significance in clay weathering. Soil Sci., 137: 216-226, 1984. 
SOON, Y.K. Fractionation of extractable aluminum in acid soils: A review and a proposed procedure. Comm. Soil Sci. Plant Anal., 24:1683-1708, 1993.

SUGUIO, K. \& MARTIN, L. Quaternary marine formations of the State of São Paulo and southern Rio de Janeiro. In: INTERNATIONAL SYMPOSIUM ON COASTAL EVOLUTION IN THE QUATERNARY, 1978, São Paulo. Anais... São Paulo, IGCB/IG-USP; SBG, 1978. 55p. (Special Publication, 1)

SUGUIO, K.; TATUMI, S.H. \& KOWATA, E.A. The Comprida Island inactive dune ridges and their possible significance for the island evolution during the Holocene, State of São Paulo, Brazil. An. Acad. Bras. Ci., 71:623-630, 1999.
TAKAHASHI, T.; FUKUOKA, T. \& DAHLGREN, R.A. Aluminum solubility and release from soil horizons dominated by aluminum-humus complexes. Soil Sci. Plant Nutr., 41:119-131, 1995.

URRUTIA, M.; MACÍAS, F. \& GARCÍA-RODEJA, E. Evaluación del $\mathrm{CuCl}_{2}$ y del $\mathrm{LaCl}_{3}$ como extractantes de aluminio en suelos ácidos de Galicia. Nova Acta Cient. Comp. (Bioloxía), 5:173-182, 1995. 